\title{
ORNL ISOTOPIC POWER FUELS QUARTERLY REPORT FOR PERIOD ENDING MARCH 31, 1974
}

\author{
C. L. Ottinger
}




\section{DISCLAIMER}

This report was prepared as an account of work sponsored by an agency of the United States Government. Neither the United States Government nor any agency Thereof, nor any of their employees, makes any warranty, express or implied, or assumes any legal liability or responsibility for the accuracy, completeness, or usefulness of any information, apparatus, product, or process disclosed, or represents that its use would not infringe privately owned rights. Reference herein to any specific commercial product, process, or service by trade name, trademark, manufacturer, or otherwise does not necessarily constitute or imply its endorsement, recommendation, or favoring by the United States Government or any agency thereof. The views and opinions of authors expressed herein do not necessarily state or reflect those of the United States Government or any agency thereof. 


\section{DISCLAIMER}

Portions of this document may be illegible in electronic image products. Images are produced from the best available original document. 
Printed in the United States of America. Available from

National Technical Information Service

U.S. Department of Commerce

5285 Port Royal Road, Springfield, Virginia 22151

Price: Printed Copy \$4.00; Microfiche $\$ 1.45$

This report was prepared as an account of work sponsored by the United States Government. Neither the United States nor the United States Atomic Energy Commission, nor any of their employees, nor any of their contractors, subcontractors, or their employees, makes any warranty, express or implied, or assumes any legal liability or responsibility for the accuracy, completeness or usefulness of any information, apparatus, product or process disclosed, or represents that its use would not infringe privately owned rights. 
ORNL-4978

UC-33 - Nuclear Propulsion

Systems and Aerospace Safety

Contract No. W-7405-eng-26

ISOTOPES DEVELOPMENT CENTER

ORNL ISOTOPIC POWER FUELS QUARTERLY REPORT FOR PERIOD ENDING MARCH 31, 1974

C. L. Ottinger

Isotopes Division

JUNE 1974

This report was pOT ICE

sponsored by the prepared as an account of work

the United States nithe States Government Nork

Commission nor or the United States Atomic Ener

their contractors any of their employees, nor any

makes any wars, subcontractors, or their amploy of

legal liability or

pletenabiliy or responsibility for the aceuracy any

pleteness or usefulness of any informe accuracy, com-

product or process disclosed or represen, apparatus, would not infringe privately, or represents that its use

OAK RIDGE NATIONAL LABORATORY
Oak Ridge, Tennessee 37830
Operated by
UNION CARBIDE CORPORATION
for the
U.S. ATOMIC ENERGY COMMISSION


SUMMARY . . . . . . . . . . . . . . . . . . 1

CURIUM-244 FUEL DEVELOPMENT . . . . . . . . . . . . . . . 2

${ }^{244} \mathrm{Cm}_{2} \mathrm{O}_{3}$ Compatibility Program ............... 2

Compatibility Couple Tests . . . . . . . . . . . 2

FY 1974 Compatibility Matrix . . . . . . . . . . . 5

Thermodynamic Calculations . . . . . . . . . . 5

${ }^{244} \mathrm{Cm}_{2} \mathrm{O}_{3}$ Property Characterization ............ 17

Helium Release.................. . 17

Vapor Pressure . . . . . . . . . . . . 24

Dimensional Stability . . . . . . . . . . . . 28

Solubility of Stored Curium Oxide

Powder in Air-Saturated Distilled Water . . . . . . . 28

Criticality of ${ }^{244} \mathrm{Cm}_{2} \mathrm{O}_{3}$ Fuels ... . . . . . . . . 29

Effects of Plutonium In-Growth ............ 32

General Operations................ 33

Curium-244 Recovery Development . . . . . . . . . . 33

REFERENCES ......................... 35

\section{LIST OF FIGURES}

Fig. 1. Helium Release Rate vs Time for ${ }^{244} \mathrm{Cm}_{2} \mathrm{O}_{3}$ Samples . . . . 18

Fig. 2. ${ }^{244} \mathrm{Cm}_{2} \mathrm{O}_{3}$ Pellets After Helium Release Experiment . . . . 20

Fig. 3. Helium Diffusion Parameters for ${ }^{244} \mathrm{Cm}_{2} \mathrm{O}_{3}$. . . . . . 21

Fig. 4. Isochronal Anneal Experiments for ${ }^{244} \mathrm{Cm}_{2} \mathrm{O}_{3}$....... 23

Fig. 5. Vapor Pressure of $\mathrm{Cm}_{2} \mathrm{O}_{3}$. . . . . . . . . . . 25

Fig. 6. Solution of $\mathrm{CmO}_{\mathrm{x}}$. . . . . . . . . . . . 28 
Table 1. Record of FY 1973 Compatibility Matrix Exposures . . . 3

Table 2. Summary of Results on Control Tests for

${ }^{24{ }^{4}} \mathrm{Cm}_{2} \mathrm{O}_{3}$ Compatibility Tests........... 4

Table 3. Partial Molar Free Energies of Mixing of

the Constituents in Hastelloy $\mathrm{C}-276$. . . . . . . 6

Table 4. Gibbs Free Energy of Formation of Oxides Involved

in Hastelloy C-276/Curium Fuel Reactions ....... 7

Table 5. Free Energies of Reaction for

Hastelloy $\mathrm{C}-276 / \mathrm{Cm}_{2} \mathrm{O}_{3}$ Reactions . . . . . . . . . 8

Table 6. Free Energies of Reaction for

Hastelloy $\mathrm{C}-276 / \mathrm{PuO}_{2}$ Reactions ............ 9

Table 7. Partial Molar Free Energies of Mixing of

the Components of $W-26 \%$ Re Alloy ........... 9

Table 8. Gibbs Free Energy of Formation of Oxides Involved in W-26\% Re Alloy/Curium Fuel Reactions . . . . . . 10

Table 9. Gibbs Free Energy of Reaction for Eqs. 25-28 . . . . . 10

Table 10. Partial Molar Free Energies of Mixing of the Components of Mo-46\% Re Alloy . . . . . . . . 11

Table 11. Gibbs Free Energies of Formation of $\mathrm{MoO}_{2}, \mathrm{ReO}_{2}, \mathrm{Pu}_{2} \mathrm{O}_{3}$, and $\mathrm{PuO}_{2}$............ 11

Table 12. Gibbs Free Energy of Reaction for Eqs. 29-32 . . . . 12

Table 13. Partial Molar Free Energies of Mixing of the Constituents in Haynes 25 Alloy . . . . . . . . . 12

Table 14. Gibbs Free Energy of Formation of Oxides Involved in Haynes 25/Curium Fuel Reactions . . . . . . . 13

Table 15. Free Energies of Reaction for Haynes $25 / \mathrm{Cm}_{2} \mathrm{O}_{3}$ Reactions 14

Table 16. Free Energies of Reaction for Haynes $25 / \mathrm{PuO}_{2}$ Reactions 14

Table 17. Partial Molar Free Energies of Mixing of Components of $\mathrm{Pt}-26 \% \mathrm{Rh}-8 \% \mathrm{~W}-0.5 \% \mathrm{Ti}$. . . . . . 15

Table 18. Gibbs Free Energy of Formation of $\mathrm{Pt}_{3} \mathrm{O}_{4}, \mathrm{Rh}_{2} \mathrm{O}, \mathrm{WO}_{2}, \mathrm{TiO}, \mathrm{Pu}_{2} \mathrm{O}_{3}$, and $\mathrm{PuO}_{2} \ldots . . . . .15$ 
Table 19. Gibbs Free Energy of Reaction for Eqs. 53-58 . . . . 16

Table 20. Gibbs Free Energy of Formation of

$\mathrm{Si}_{3} \mathrm{~N}_{4}, \mathrm{CeN}, \mathrm{SiO}_{2}, \mathrm{Pu}_{2} \mathrm{O}_{3}$, and $\mathrm{PuO}_{2}$......... 16

Table 21. Gibbs Free Energy for Eqs. 59 and 60 . . . . . . . 17

Table 22. Mass and Dimensional Data . . . . . . . . 18

Table 23. Helium Inventory Measurements . . . . . . . . 21

Table 24. Experimental Conditions of the Isochronal

Anneal Experiments for ${ }^{244} \mathrm{Cm}_{2} \mathrm{O}_{3}$.......... 22

Table 25. Effect of the Nature of Target Surface on

the Amount of Curium-244 Collected ........ 26

Table 26. Curium-244 Heat Source Descriptions . . . . . . . 29

Table 27. Results of Criticality Evaluation of Curium-244 Sources 31 
ORNL ISOTOPIC POWER FUELS QUARTERLY REPORT FOR PERIOD ENDING MARCH 37, 1974

C. L. Ottinger

\begin{abstract}
SUMMARY
Examination of the 49 compatibility test couples which were taken off test in December 1973 was started. Nonradioactive control couples representative of these 49 specimens were examined. Development of the techniques required for fabrication of the 11-couple combined compatibility/tensile strength test matrix continued. Free energy calculations were made covering possible reactions of ${ }^{2}{ }^{4} \mathrm{Cm}_{2} \mathrm{O}_{3}$ fuel with six potential encapsulants.
\end{abstract}

Helium release experiments at 500,650 , and $800^{\circ} \mathrm{C}$ were completed. These tests indicated that the controlling mechanism for helium release from ${ }^{244} \mathrm{Cm}_{2} \mathrm{O}_{3}$ is different at different temperatures, with a change in mechanism occurring in the region of $600-700^{\circ} \mathrm{C}$. Vapor pressure measurements were made on ${ }^{244} \mathrm{Cm}_{2} \mathrm{O}_{3}$. A $25-\mathrm{W}{ }^{24{ }^{4}} \mathrm{Cm}_{2} \mathrm{O}_{3}$ pellet was exposed to dry air; it exhibited a similar edge-fracturing effect as that observed in a previous test. A leach rate study was done on a sample of airoxidized ${ }^{244} \mathrm{Cm}_{2} \mathrm{O}_{3}$ fuel.

Evaluation of the criticality aspects of three reference ${ }^{244} \mathrm{Cm}$-fueled source assemblies was completed; the results indicated that no criticality problems would be expected. A re-orientation of the ${ }^{244} \mathrm{Cm}$ development program from emphasis on experimental properties measurements to emphasis on laboratory-scale development required some changes in the utilization of facilities and modification of some experimental plans. Plans were initiated for a ${ }^{244} \mathrm{Cm}$ recovery process development experimental program. 


\author{
CURIUM-244 FUEL DEVELOPMENT \\ (Division of Space Nuclear Systems Program LR 300103 3) \\ ${ }^{244} \mathrm{Cm}_{2} \mathrm{O}_{3}$ Compatibility Program
}

Compatibility Couple Tests (T. A. Butzer and J.R. DiStefano)

Examination of the compatibility couples from the FY 1973 matrix shown in Table 1 was delayed for approximately two months while the examination facility was shut down for equipment reworking and maintenance. The nonradioactive control exposures for the 900 and $1400^{\circ} \mathrm{C}$ tests were completed, and the specimens were examined. A summary of the results of the 900 and $1400^{\circ} \mathrm{C}$ control test examinations is given in Table 2. Some conclusions that can be drawn are (1) the precious metals were unaffected by exposure to helium, vacuum, or graphite under the test conditions;

(2) the superalloys reacted with graphite at $900^{\circ} \mathrm{C}$ but did not bond to the graphite when in contact; (3) molybdenum and tantalum reacted with graphite at $1400^{\circ} \mathrm{C}$ when there was surface contact; and (4) the molybdenum/graphite reaction was worse than the tantalum/graphite reaction, with both reactions worse under vacuum conditions than under helium.

After completion of the maintenance work at the examination facility in March, examination of the couples from sets $1,2,7,8,9$, and 10 (see Table 1) was resumed. The noble metals and noble metal alloys from sets 1 and 2 were selected for examination on a first priority basis. Top disk samples were removed for chemical analysis; the results are shown below:

\begin{tabular}{lcc} 
& \multicolumn{2}{c}{ Oxygen Concentration } \\
\cline { 2 - 3 } Material & Set 1 & Set 2 \\
\cline { 2 - 3 } Iridium & $<10$ & $<10$ \\
Platinum & $<10$ & $<10$ \\
Pt-20\% Rh & $<10$ & $<10$ \\
Pt-26\% Rh-8\% W & 24 & 17
\end{tabular}

Only the $\mathrm{Pt}-26 \% \mathrm{Rh}-8 \% \mathrm{~W}(\mathrm{Pt}-2608)$ alloy picked up oxygen. In the $1100^{\circ} \mathrm{C}$ tests, $\mathrm{Pt}-2608 \mathrm{M}$ (containing $0.5 \% \mathrm{Ti}$ ) had been found to pick up oxygen $(23-107 \mathrm{ppm}) .1$

Preliminary examination of the samples from sets 1 and 2 indicated that the unalloyed platinum reacted slightly with the $\mathrm{Cm}_{2} \mathrm{O}_{3}$, but the other materials appeared to be unaffected. At $1100^{\circ} \mathrm{C}$ unalloyed platinum reacted with $\mathrm{Cm}_{2} \mathrm{O}_{3}$ to form a $\mathrm{Pt}-\mathrm{Cm}$ compound, ${ }^{\mathrm{l}}$ but little or no interaction occurred between the fuel and the other test materials ( $\mathrm{Pt}-20 \% \mathrm{Rh}$ was not tested at $1100^{\circ} \mathrm{C}$ ). 
Table 1. Record of FY 1973 Compatibility Matrix Exposures

\begin{tabular}{rllcc}
\hline Set $^{a}$ & $\begin{array}{c}\text { Start } \\
\text { of Test }\end{array}$ & $\begin{array}{c}\text { Thermal } \\
\text { Cycles }\end{array}$ & $\begin{array}{c}\text { Cumulative } \\
\text { Hours }\end{array}$ & End of Test \\
\hline 1 & $5-3-73$ & 1 & 5021 & $12-3-73$ \\
2 & $5-3-73$ & 1 & 5021 & $12-3-73$ \\
3 & $4-16-73$ & 3 & 2560 & $8-6-73$ \\
4 & $4-16-73$ & 3 & 2560 & $8-6-73$ \\
5 & $4-17-73$ & 2 & 2510 & $8-9-73$ \\
6 & $4-17-73$ & 2 & 2510 & $8-9-73$ \\
7 & $5-3-73$ & 2 & 4892 & $11-27-73$ \\
8 & $5-3-73$ & 2 & 4892 & $11-27-73$ \\
9 & $5-3-73$ & 2 & 5016 & $12-4-73$ \\
10 & $5-3-73$ & 2 & 5016 & $12-4-73$ \\
\hline
\end{tabular}

$a_{\text {Set identifications: }}$

1. $900^{\circ} \mathrm{C} ; 5000 \mathrm{hr}$; helium atmosphere; Ir, C, Pt, Hf-1\% Pt$0.5 \% \mathrm{Pd}$, Hastelloy $\mathrm{C}-276$, Haynes 25 , Haynes $188, \mathrm{ThO}_{2}$, $\mathrm{Pt}-20 \% \mathrm{Rh}$, and Pt $-26 \% \mathrm{Rh}-8 \% \mathrm{~W}$.

2. $900^{\circ} \mathrm{C}$; $5000 \mathrm{hr}$; graphite, helium atmosphere; Ir, Pt, Hf $-1 \% \mathrm{Pt}-0.5 \% \mathrm{Pd}$, Hastelloy C-276, Haynes 25, Haynes 188, $\mathrm{ThO}_{2}, \mathrm{Pt}-20 \% \mathrm{Rh}$, and Pt $-26 \% \mathrm{Rh}-8 \% \mathrm{~W}$.

3. $1100^{\circ} \mathrm{C} ; 2500 \mathrm{hr}$; graphite, helium atmosphere; Ir, Pt, $\mathrm{Pt}_{3} \mathrm{Ir}$, and $\mathrm{Pt}-26 \% \mathrm{Rh}-8 \% \mathrm{~W}-0.5 \% \mathrm{Ti}$.

4. $1100^{\circ} \mathrm{C} ; 2500 \mathrm{hr}$; dynamic vacuum; Ir, $\mathrm{Pt}, \mathrm{Pt}_{3} \mathrm{Ir}, \mathrm{C}$, and Pt-26\% Rh-8\% W-0.5\% Ti.

5. $1100^{\circ} \mathrm{C} ; 2500 \mathrm{hr}$; helium atmosphere; Ir, Pt, Pt 3 Ir, C, and $\mathrm{Pt}-26 \% \mathrm{Rh}-8 \% \mathrm{~W}-0.5 \% \mathrm{Ti}$.

6. $1100^{\circ} \mathrm{C} ; 2500 \mathrm{hr}$; graphite, dynamic vacuum; Ir, Pt, $\mathrm{Pt} \mathrm{I}_{3} \mathrm{Ir}$, and $\mathrm{Pt}-26 \% \mathrm{Rh}-8 \% \mathrm{~W}-0.5 \% \mathrm{Ti}$.

7. $1400^{\circ} \mathrm{C}$; $5000 \mathrm{hr}$; graphite, helium atmosphere, Ir, C, Mo, $\mathrm{Mo}-46 \% \mathrm{Re}, \mathrm{Ta}, \mathrm{T}-111, \mathrm{~W}$, and $\mathrm{W}-26 \% \mathrm{Re}$.

8. $1400^{\circ} \mathrm{C} ; 5000 \mathrm{hr}$; dynamic vacuum; Ir, Mo, Mo-46\% Re, Ta, $\mathrm{T}-111, \mathrm{~W}$, and $\mathrm{W}-26 \% \mathrm{Re}$.

9. $1400^{\circ} \mathrm{C}$; $5000 \mathrm{hr}$; helium atmosphere; Ir, Mo, Mo-46\% Re, $\mathrm{Ta}, \mathrm{T}-111, \mathrm{~W}$, and $\mathrm{W}-26 \% \mathrm{Re}$.

10. $1400^{\circ} \mathrm{C} ; 5000 \mathrm{hr}$; graphite, dynamic vacuum; Ir, C, Mo, $\mathrm{Mo}-46 \% \mathrm{Re}, \mathrm{Ta}, \mathrm{T}-111, \mathrm{~W}$, and $\mathrm{W}-26 \% \mathrm{Re}$. 
Table 2. Summary of Results on Control Tests for ${ }^{24{ }^{4}} \mathrm{Cm}_{2} \mathrm{O}_{3}$ Compatibility Tests

\begin{tabular}{|c|c|c|c|}
\hline \multirow{2}{*}{ Material } & \multicolumn{2}{|c|}{ Visual Observations } & \multirow{2}{*}{$\begin{array}{l}\text { Weight } \\
\text { Change (g) }\end{array}$} \\
\hline & Capsule & Specimen & \\
\hline Conditions: & $900^{\circ} \mathrm{C} ; 5000 \mathrm{hr} ;$ static helium; $\mathrm{Al}_{2} \mathrm{O}_{3}$ & capsule holder: Hastelloy $\mathrm{X}$ outer container & \\
\hline Hastelloy $\mathrm{C}-276$ & $\begin{array}{l}\text { Gray-black-gold film on outer surface, } \\
\text { especially on end touching } \mathrm{Al}_{2} \mathrm{O}_{3}\end{array}$ & Du11 & 0 \\
\hline Haynes 188 & $\begin{array}{l}\text { Same as above, except gold film on } \\
\text { top end not touching } \mathrm{Al}_{2} \mathrm{O}_{3}\end{array}$ & Du11 & 0 \\
\hline Haynes 25 & Same as Hastelloy $\mathrm{C}-276$ & Dul1 & 0 \\
\hline $\mathrm{HF}-1 \% \mathrm{Pt}-0.5 \% \mathrm{Pd}$ & $\begin{array}{l}\text { Du11, alumina-colored layer on outside } \\
\text { surface, gold-colored layer below }\end{array}$ & $\begin{array}{l}\text { Dul1, black on surface touching } \\
\text { capsule bottom }\end{array}$ & -0.0021 \\
\hline Graphite & $\begin{array}{l}\text { No change on outside, but metallic } \\
\text { layer on inside }\end{array}$ & $\begin{array}{l}\text { Metallic layer on all surfaces except } \\
\text { bottom where in contact with graphite } \\
\text { capsule }\end{array}$ & 0 \\
\hline $\mathrm{Pt}-20 \% \mathrm{Rh}$ & Slight dull appearance on outside & $\begin{array}{l}\text { Bright, except bottom surface } \\
\text { touching capsule }\end{array}$ & 0 \\
\hline $\mathrm{Pt}-26 \% \mathrm{Rh}-8 \% \mathrm{~W}$ & Slight dull appearance on outside & Bright & 0 \\
\hline Conditions: & $\begin{array}{l}900^{\circ} \mathrm{C} ; 5000 \mathrm{hr} \text { static helium; graphi } \\
\text { graphite capsule holder; Hastelloy X }\end{array}$ & $\begin{array}{l}\text { ite intermediate capsule; } \\
\text { outer container }\end{array}$ & \\
\hline Hastelloy $\mathrm{C}-276$ & Gray-black layer on outer surface & Black & +0.0008 \\
\hline Haynes 188 & Gray-black layer on outer surface & Black & +0.0006 \\
\hline Haynes 25 & Light-gray layer on outer surface & Black & +0.0007 \\
\hline $\mathrm{Hf}-1 \% \mathrm{Pt}-0.5 \% \mathrm{Pd}$ & Gray-gold layer on outer surface & Du11, very light gold layer in some areas & 0 \\
\hline $\mathrm{ThO}_{2}$ & Surface spotted gray-black-white & Surface spotted gray-black-white & 0 \\
\hline Platinum & Etched appearance & Bright, but etched appearance & 0 \\
\hline $\mathrm{Pt}-20 \% \mathrm{Rh}$ & Bright & Du11 & 0 \\
\hline $\mathrm{Pt}-26 \% \mathrm{Rh}-8 \% \mathrm{~W}$ & Bright & Bright, one dark spot & 0 \\
\hline Iridium & Bright & Bright & 0 \\
\hline
\end{tabular}

Conditions: $1400^{\circ} \mathrm{C}$; $5000 \mathrm{hr}$; static helium; graphite intermediate capsule; graphite capsule holder; tantalum outer container

\begin{tabular}{|c|c|c|c|}
\hline Molybdenum & $\begin{array}{l}\text { Bonded to graphite intermediate } \\
\text { capsule, could not be separated }\end{array}$ & Etched but bright appearance & 0 \\
\hline Tantalum & Dull on outer surface & Bright, large grains visible & 0 \\
\hline $\mathrm{T}-111$ & Dull on outer surface & Du11. & 0 \\
\hline Tungsten & Shiny & Bright & a \\
\hline W-26\% Re & Shiny & Dull to shiny & a \\
\hline Iridium & Shiny, etched & Bright, etched & a \\
\hline Graphite & Light gray & No change & 0 \\
\hline \multicolumn{4}{|c|}{$\begin{array}{l}\text { Conditions: } \begin{array}{l}1400^{\circ} \mathrm{C}, 5000 \mathrm{hr} \text {; drnamic vacuum; graphite intermediate capsule; } \\
\text { graphite capsule holder; tantalum outer container }\end{array} \\
\end{array}$} \\
\hline Molybdenum & $\begin{array}{l}\text { Bonded to graphite intermediate cap- } \\
\text { sule; inside surface irregular; } \\
\text { graphite appeared to have penetrated } \\
\text { to inside; capsule broke when we } \\
\text { attempted to remove specimen; } \\
\text { cleavage-type fracture }\end{array}$ & $\begin{array}{l}\text { Could not be removed; reacted } \\
\text { with graphite and capsule }\end{array}$ & a \\
\hline $\operatorname{Mo}-46 \% \operatorname{Re}$ & Bonded to graphite; could not remove & Bright & +0.0014 \\
\hline Tantalum & Bonded to graphite; could not remove & Bright & 0 \\
\hline $\mathrm{T}-111$ & Shiny & Shiny & 0 \\
\hline Tungsten & Bright & Bright & $\mathbf{a}$ \\
\hline$W-26 \% \mathrm{Re}$ & Dull to shiny & Shiny & 0 \\
\hline Iridium & Bright, etched & Stuck to capsule; could not remove; shiny & $\mathbf{a}$ \\
\hline Graphite & Unchanged & Unchanged & 0 \\
\hline
\end{tabular}

a Not obtained; specimen damaged when capsule was opened. 
FY 1974 Compatibility Matrix (J. R. Distefano and C. L. Ottinger)

The materials and exposure conditions for the FY 1974 compatibility matrix are shown below:

\begin{tabular}{|c|c|c|}
\hline $\begin{array}{c}5000 \mathrm{hr}, 900^{\circ} \mathrm{C} \\
\text { Helium } \\
\end{array}$ & $\begin{array}{c}5000 \mathrm{hr}, 1100^{\circ} \mathrm{C} \\
\text { Vacuum } \\
\end{array}$ & $\begin{array}{c}10,000 \text { hr, } 1400^{\circ} \mathrm{C} \\
\text { Vacuum } \\
\end{array}$ \\
\hline $\begin{array}{ll}\text { Haynes } & 188 \\
\mathrm{Ta}-10 \% & \mathrm{~W} \\
\mathrm{Mo}-46 \% & \mathrm{Re} \\
\mathrm{TZM} & \\
\mathrm{ZrO}_{3} & \\
\mathrm{Si}_{3} \mathrm{~N}_{4} & \end{array}$ & $\begin{array}{l}\text { Pt }-30 \% \text { Rh- } 8 \% \mathrm{~W} \\
\mathrm{Ta}-10 \% \mathrm{~W} \\
\mathrm{Mo}-46 \% \text { Re }\end{array}$ & $\begin{array}{l}\text { Mo-46\% Re } \\
W-26 \% \operatorname{Re}\end{array}$ \\
\hline
\end{tabular}

Except for the $\mathrm{ZrO}_{2}$ and $\mathrm{Si}_{3} \mathrm{~N}_{4}$, these test couples will include specimens which will be used to test tensile strength. The tensile strength specimens will be in contact with ${ }^{244} \mathrm{Cm}_{2} \mathrm{O}_{3}$ fuel pieces in the form of flat bars (approximately $0.5 \times 0.125 \times 0.050 \mathrm{in}$. ). The $\mathrm{ZrO}_{2}$ and $\mathrm{Si}_{3} \mathrm{~N}_{4}$ couples will use cylindrical pellets and disk-shaped specimens.

A11 the required materials for this matrix were received from suppliers. Material and labor to produce the finished test specimens of $\mathrm{Ta}-10 \% \mathrm{~W}$, Haynes 188, TZM, and W-26\% Re were provided by the Metals Division of the Thermo Electron Company; the $\mathrm{Si}_{3} \mathrm{~N}_{4}$ materials were provided by General Atomic. No reasonable bids for fabrication of the $\mathrm{ZrO}_{2}$ specimens were received from commercial vendors, so the design was modified so that commercially available $\mathrm{ZrO}_{2}$ inserts will be used in graphite outer containers. The Mo-46\% Re and $\mathrm{Pt}-30 \% \mathrm{Rh}-8 \% \mathrm{~W}$ were provided from ORNL stocks. Fabrication of the tensile strength specimens was started.

The rectangular ${ }^{244} \mathrm{Cm}_{2} \mathrm{O}_{3}$ test fuel form presents an unusual in-cell fabrication problem. The procedure currently being tested involves a slotted die and punch assembly which is retained in a graphite adapter during hot pressing. After some technique development, pressings of $\mathrm{Gd}_{2} \mathrm{O}_{3}$ were done successfully in cold runs; demonstration runs with ${ }^{244} \mathrm{Cm}_{2} \mathrm{O}_{3}$ were scheduled for April. The main expected problem is the removal of the ${ }^{244} \mathrm{Cm}_{2} \mathrm{O}_{3}$ piece from the slotted die. Assembly of the compatibility couples is scheduled for late May.

\section{Thermodynamic Calculations (E. E. Ketchen)}

Calculational studies of potential reactions of encapsulant materials with ${ }^{244} \mathrm{Cm}_{2} \mathrm{O}_{3}$ fuels were continued. Six materials, five alloys and $\mathrm{Si}_{3} \mathrm{~N}_{4}$, were investigated from a thermodynamic viewpoint. Samples of all of these materials are currently being tested in the FY 1973 compatibility matrix or are scheduled for testing in the FY 1974 matrix. In studies of the alloys, common assumptions were that there would be no compound formation between constituents, that the most favorable oxides of the alloy constituents would be formed, and that oxygen solubility in the alloy would not be considered. Since free energy values for $\mathrm{Cm}_{2} \mathrm{O}_{3}$ 
are not available, the values for $\mathrm{Pu}_{2} \mathrm{O}_{3}$ were used as stand-ins. Partial molar free energy calculations were made by the method described previously. ${ }^{1}$ Free energies of formation were calculated from the data of Glassner ${ }^{2}$ and oetting. ${ }^{3}$ Since possible reactions with $\mathrm{Si}_{3} \mathrm{~N}_{4}$ involve products other than oxides, a different approach was used; this is described in detail under "Silicon Nitride" below. A reference fuel consisting of $95 \% \mathrm{Cm}_{2} \mathrm{O}_{3}$ and $5 \% \mathrm{PuO}_{2}$ was assumed in all cases.

Reactions With Hastelloy C-276 - The composition of Hastelloy $\mathrm{C}-276$ in atom percent is: $\mathrm{Ni}, 57.95 ; \mathrm{Cr}, 19.21 ; \mathrm{Mo}, 10.41 ; \mathrm{Fe}, 6.15 ; \mathrm{W}, 1.36$; Co, $2.65 ; \mathrm{Mn}, 1.14 ; \mathrm{V}, 0.37 ; \mathrm{C}, 0.52 ; \mathrm{Si}, 0.11 ; \mathrm{P}, 0.081$; and $\mathrm{S}, 0.058$. The calculated partial molar free energies of mixing for these constituents from 600 to $1300^{\circ} \mathrm{C}$ are given in Table 3 .

Table 3. Partial Molar Free Energies of Mixing of the Constituents in Hastelloy $\mathrm{C}-276$

\begin{tabular}{lrrrrr}
\hline \multirow{2}{*}{ Components } & \multicolumn{5}{c}{$-\Delta \bar{G}_{\mathrm{m}}(\mathrm{ca} 1 / \mathrm{mo} \mathrm{le})$} \\
\cline { 2 - 6 } & $600^{\circ} \mathrm{C}$ & $800^{\circ} \mathrm{C}$ & $1000^{\circ} \mathrm{C}$ & $1200^{\circ} \mathrm{C}$ & $1300^{\circ} \mathrm{C}$ \\
\hline $\mathrm{Ni}$ & 920 & 1,130 & 1,350 & 1,570 & 1,670 \\
$\mathrm{Cr}$ & 2,620 & 3,280 & 3,930 & 4,590 & 4,920 \\
$\mathrm{Mo}$ & 3,880 & 4,780 & 5,680 & 6,580 & 7,030 \\
$\mathrm{Fe}$ & 4,830 & 5,940 & 7,050 & 8,160 & 8,710 \\
$\mathrm{~W}$ & 7,380 & 9,090 & 10,750 & 12,510 & 13,360 \\
$\mathrm{Co}$ & 6,300 & 7,740 & 9,190 & 10,630 & 11,350 \\
$\mathrm{Mn}$ & 7,710 & 9,490 & 11,270 & 13,050 & 13,940 \\
$\mathrm{~V}$ & 9,730 & 11,960 & 14,180 & 16,410 & 17,530 \\
$\mathrm{C}$ & 9,030 & 11,120 & 13,210 & 15,300 & 16,350 \\
$\mathrm{Si}$ & 11,790 & 14,490 & 17,200 & 19,900 & 21,250 \\
$\mathrm{P}$ & 12,340 & 15,170 & 18,000 & 20,830 & 22,690 \\
$\mathrm{~S}$ & 12,880 & 15,840 & 18,800 & 21,760 & 23,240 \\
& & & & & \\
\hline
\end{tabular}

The free energies of formation for the oxides considered in the ${ }^{244} \mathrm{Cm}_{2} \mathrm{O}_{3}$ Fuel/Hastelloy C-276 reaction calculations are given in Table 4 . 
Tab1e 4. Gibbs Free Energy of Formation of Oxides Involved in Hastelloy C-276/Curium Fuel Reactions

\begin{tabular}{lrrrrr}
\hline \multirow{2}{*}{ Constituents } & \multicolumn{5}{c}{$\Delta \mathrm{G}_{\mathrm{f}}(\mathrm{kcal} / \mathrm{mole})$} \\
\cline { 2 - 6 } & $600^{\circ} \mathrm{C}$ & $800^{\circ} \mathrm{C}$ & $1000^{\circ} \mathrm{C}$ & $1200^{\circ} \mathrm{C}$ & $1300^{\circ} \mathrm{C}$ \\
\hline $\mathrm{NiO}$ & 39.06 & 35.01 & 31.01 & 27.10 & 25.15 \\
$\mathrm{Cr}_{2} \mathrm{O}_{3}$ & 203.92 & 189.81 & 175.72 & 161.67 & 154.63 \\
$\mathrm{MoO}_{2}$ & 95.60 & 87.91 & 80.36 & 72.94 & 69.27 \\
$\mathrm{Fe}_{3} \mathrm{O}_{4}$ & 199.79 & 185.20 & 170.88 & 156.61 & 149.47 \\
$\mathrm{WO}_{2}$ & 101.16 & 93.85 & 86.78 & 79.96 & 76.80 \\
$\mathrm{CoO}$ & 39.46 & 35.37 & 31.02 & 27.08 & 24.96 \\
$\mathrm{MnO}$ & 76.82 & 73.30 & 69.76 & 66.23 & 64.27 \\
$\mathrm{~V}_{2} \mathrm{O}_{3}$ & 242.27 & 230.90 & 219.82 & 209.03 & 203.72 \\
$\mathrm{CO}_{2}$ & 94.55 & 94.36 & 94.24 & 94.02 & 94.00 \\
$\mathrm{SiO}_{2}$ & 172.92 & 164.60 & 156.33 & 148.10 & 144.00 \\
$\mathrm{P}_{2} \mathrm{O}_{3}$ & 212.19 & 200.88 & 189.69 & 178.62 & 173.16 \\
$\mathrm{SO}_{2}$ & 65.74 & 63.80 & 61.86 & 59.93 & 58.97 \\
$\mathrm{Pu}_{2} \mathrm{O}_{3}$ & 352.12 & 339.32 & 326.94 & 314.98 & 309.15 \\
$\mathrm{PuO}_{2}$ & 215.55 & 207.05 & 198.70 & 190.53 & 186.50 \\
\hline
\end{tabular}

The free energies from Tables 3 and 4 were used to calculate the free energies for the reactions given in Eqs. 1-24 below.

$$
\begin{aligned}
& 3 \mathrm{Ni} \text { (in Hastelloy } \mathrm{C}-276)(\mathrm{s})+\mathrm{Cm}_{2} \mathrm{O}_{3}(\mathrm{~s}) \rightarrow 3 \mathrm{NiO}(\mathrm{s})+2 \mathrm{Cm}(\mathrm{s}) \\
& \mathrm{Ni} \text { (in Hastelloy } \mathrm{C}-276)(\mathrm{s})+2 \mathrm{PuO}_{2}(\mathrm{~s}) \rightarrow \mathrm{NiO}(\mathrm{s})+\mathrm{Pu}_{2} \mathrm{O}_{3}(\mathrm{~s}) \\
& 2 \mathrm{Cr} \text { (in Hastelloy } \mathrm{C}-276)(\mathrm{s})+\mathrm{Cm}_{2} \mathrm{O}_{3}(\mathrm{~s}) \rightarrow \mathrm{Cr}_{2} \mathrm{O}_{3}(\mathrm{~s})+2 \mathrm{Cm}(\mathrm{s}) \\
& 2 \mathrm{Cr} \text { (in Hastelloy } \mathrm{C}-276)(\mathrm{s})+6 \mathrm{PuO}_{2}(\mathrm{~s}) \rightarrow \mathrm{Cr}_{2} \mathrm{O}_{3}(\mathrm{~s})+3 \mathrm{Pu}_{2} \mathrm{O}_{3}(\mathrm{~s}) \\
& 3 \mathrm{Mo}(\text { in Hastelloy } \mathrm{C}-276)(\mathrm{s})+2 \mathrm{Cm}_{2} \mathrm{O}_{3}(\mathrm{~s}) \rightarrow 3 \mathrm{MoO}_{2}(\mathrm{~s})+4 \mathrm{Cm}(\mathrm{s}) \\
& \mathrm{Mo}(\text { in Hastelloy } \mathrm{C}-276)(\mathrm{s})+4 \mathrm{PuO}_{2}(\mathrm{~s}) \rightarrow \mathrm{MoO}_{2}(\mathrm{~s})+2 \mathrm{Pu}_{2} \mathrm{O}_{3}(\mathrm{~s}) \\
& 9 \mathrm{Fe} \text { (in Hastelloy } \mathrm{C}-276)(\mathrm{s})+4 \mathrm{Cm}_{2} \mathrm{O}_{3}(\mathrm{~s}) \rightarrow 3 \mathrm{Fe}_{3} \mathrm{O}_{4}(\mathrm{~s})+8 \mathrm{Cm}(\mathrm{s}) \\
& 3 \mathrm{Fe}(\text { in Hastelloy } \mathrm{C}-276)(\mathrm{s})+8 \mathrm{PuO}_{2}(\mathrm{~s}) \rightarrow \mathrm{Fe}_{3} \mathrm{O}_{4}(\mathrm{~s})+4 \mathrm{Pu}_{2} \mathrm{O}_{3}(\mathrm{~s}) \\
& 3 \mathrm{~W}(\text { in Hastelloy } \mathrm{C}-276)(\mathrm{s})+2 \mathrm{Cm}_{2} \mathrm{O}_{3}(\mathrm{~s}) \rightarrow 3 \mathrm{WO}_{2}(\mathrm{~s})+4 \mathrm{Cm}(\mathrm{s}) \\
& \mathrm{W}(\text { in Hastelloy } \mathrm{c}-276)(\mathrm{s})+4 \mathrm{PuO}_{2}(\mathrm{~s}) \rightarrow \mathrm{WO}_{2}(\mathrm{~s})+2 \mathrm{Pu}_{2} \mathrm{O}_{3}(\mathrm{~s}) \\
& 3 \mathrm{Co}(\text { in Hastelloy } \mathrm{C}-276)(\mathrm{s})+\mathrm{Cm}_{2} \mathrm{O}_{3}(\mathrm{~s}) \rightarrow 3 \mathrm{CoO}(\mathrm{s})+2 \mathrm{Cm}(\mathrm{s}) \\
& \mathrm{Co}(\text { in Hastelloy } \mathrm{C}-276)(\mathrm{s})+2 \mathrm{PuO}_{2}(\mathrm{~s}) \rightarrow \mathrm{CoO}(\mathrm{s})+\mathrm{Pu}_{2} \mathrm{O}_{3}(\mathrm{~s}) \\
& 3 \mathrm{Mn} \text { (in Hastelloy } \mathrm{C}-276)(\mathrm{s})+\mathrm{Cm}_{2} \mathrm{O}_{3}(\mathrm{~s}) \rightarrow 3 \mathrm{Mno}(\mathrm{s})+2 \mathrm{Cm}(\mathrm{s}) \\
& \mathrm{Mn}(\text { in Hastelloy } \mathrm{C}-276)(\mathrm{s})+2 \mathrm{PuO}_{2}(\mathrm{~s}) \rightarrow \mathrm{MnO}(\mathrm{s})+\mathrm{Pu}_{2} \mathrm{O}_{3}(\mathrm{~s}) \\
& 2 \mathrm{~V} \text { (in Hastelloy } \mathrm{C}-276)(\mathrm{s})+\mathrm{Cm}_{2} \mathrm{O}_{3}(\mathrm{~s}) \rightarrow \mathrm{V}_{2} \mathrm{O}_{3}(\mathrm{~s})+2 \mathrm{Cm}(\mathrm{s}) \\
& 2 \mathrm{~V}(\text { in Hastelloy } \mathrm{C}-276)(\mathrm{s})+6 \mathrm{PuO}_{2}(\mathrm{~s}) \rightarrow \mathrm{V}_{2} \mathrm{O}_{3}(\mathrm{~s})+3 \mathrm{Pu}_{2} \mathrm{O}_{3}(\mathrm{~s}) \\
& 3 \mathrm{C}(\text { in Haste1loy } \mathrm{C}-276)(\mathrm{s})+2 \mathrm{Cm}_{2} \mathrm{O}_{3}(\mathrm{~s}) \rightarrow 3 \mathrm{CO}_{2}(\mathrm{~g})+4 \mathrm{Cm}(\mathrm{s})
\end{aligned}
$$




$$
\begin{aligned}
& \mathrm{C}(\text { in Hastelloy } \mathrm{C}-276)(\mathrm{s})+4 \mathrm{PuO}_{2}(\mathrm{~s}) \rightarrow \mathrm{CO}_{2}(\mathrm{~g})+2 \mathrm{Pu}_{2} \mathrm{O}_{3}(\mathrm{~s}) \\
& 3 \mathrm{Si}(\text { in Hastelloy } \mathrm{C}-276)(\mathrm{s})+2 \mathrm{Cm}_{2} \mathrm{O}_{3}(\mathrm{~s}) \rightarrow 3 \mathrm{SiO}_{2}(\mathrm{~s})+4 \mathrm{Cm}(\mathrm{s}) \\
& \mathrm{Si}(\text { in Hastelloy } \mathrm{C}-276)(\mathrm{s})+4 \mathrm{PuO}_{2}(\mathrm{~s}) \rightarrow \mathrm{SiO}_{2}(\mathrm{~s})+2 \mathrm{Pu}_{2} \mathrm{O}_{3}(\mathrm{~s}) \\
& 2 \mathrm{P}(\text { in Hastelloy } \mathrm{C}-276)(\mathrm{s})+\mathrm{Cm}_{2} \mathrm{O}_{3}(\mathrm{~s}) \rightarrow \mathrm{P}_{2} \mathrm{O}_{3}(\mathrm{~s})+2 \mathrm{Cm}(\mathrm{s}) \\
& 2 \mathrm{P}(\text { in Hastelloy } \mathrm{C}-276)(\mathrm{s})+6 \mathrm{PuO}_{2}(\mathrm{~s}) \rightarrow \mathrm{P}_{2} \mathrm{O}_{3}(\mathrm{~s})+3 \mathrm{Pu}_{2} \mathrm{O}_{3}(\mathrm{~s}) \\
& 3 \mathrm{~S}(\text { in Hastelloy } \mathrm{C}-276)(\mathrm{s})+2 \mathrm{Cm}_{2} \mathrm{O}_{3}(\mathrm{~s}) \rightarrow 3 \mathrm{SO}_{2}(\mathrm{~g})+4 \mathrm{Cm}(\mathrm{s}) \\
& \mathrm{S}(\text { in Hastelloy } \mathrm{C}-276)(\mathrm{s})+4 \mathrm{PuO}_{2}(\mathrm{~s}) \rightarrow \mathrm{SO}_{2}(\mathrm{~g})+2 \mathrm{Pu}_{2} \mathrm{O}_{3}(\mathrm{~s})
\end{aligned}
$$

\begin{tabular}{|c|c|c|c|c|c|}
\hline \multirow{2}{*}{ Equation } & \multicolumn{5}{|c|}{$\Delta G_{r}\left(\mathrm{cal} / \mathrm{mole} \mathrm{Cm}_{2} \mathrm{O}_{3}\right)$} \\
\hline & $600^{\circ} \mathrm{C}$ & $800^{\circ} \mathrm{C}$ & $1000^{\circ} \mathrm{C}$ & $1200^{\circ} \mathrm{C}$ & $1300^{\circ} \mathrm{C}$ \\
\hline 1 & 237,680 & 237,700 & 237,950 & 238,390 & 238,720 \\
\hline 3 & 153,450 & 156,070 & 159,090 & 162,490 & 164,360 \\
\hline 5 & 209,310 & 208,170 & 207,260 & 206,560 & 206,300 \\
\hline 7 & 213,140 & 213,790 & 214,640 & 215,870 & 216,650 \\
\hline 9 & 211,460 & 212,190 & 212,900 & 213,800 & 214,000 \\
\hline 11 & 252,650 & 256,440 & 261,430 & 265,640 & 268,320 \\
\hline 13 & 144,790 & 147,880 & 151,460 & 155,430 & 158,170 \\
\hline 15 & 129,300 & 132,330 & 135,490 & 138,770 & 140,480 \\
\hline 17 & 223,840 & 214,470 & 205,400 & 196,900 & 192,680 \\
\hline 19 & 110,420 & 114,160 & 118,240 & 122,680 & 125,020 \\
\hline 21 & $-47,590$ & $-32,090$ & $-16,430$ & -590 & 8,220 \\
\hline 23 & 272,820 & 267,370 & 262,340 & 257,720 & 255,550 \\
\hline
\end{tabular}

The results of the calculations for the reactions involving $\mathrm{Cm}_{2} \mathrm{O}_{3}$ are given in Table 5; the results for the reactions involving $\mathrm{PuO}_{2}$ are given in Table 6 .

Table 5. Free Energies of Reaction for Hastelloy $\mathrm{C}-276 / \mathrm{Cm}_{2} \mathrm{O}_{3}$ Reactions

Since the free energy of Eq. 21 is negative over the $600-1200^{\circ} \mathrm{C}$ temperature range, the reaction of $\mathrm{Cm}_{2} \mathrm{O}_{3}$ with phosphorus of the alloy would be predicted. Likewise the free energy of Eqs. 20 and 22 are negative, and the reaction of $\mathrm{PuO}_{2}$ with silicon and phosphorus of the alloy is predicted. Since phosphorus is so reactive, it is highly likely that it may be involved in a compound formation with one of the other constituents of the alloy which could change the thermodynamic picture. However, the small percentage of silicon is also expected to react. Experimental verification of the small interaction of Hastelloy $\mathrm{C}-276$ with the curium product is needed. 
Table 6. Free Energies of Reaction for Hastelloy $\mathrm{C}-276 / \mathrm{PuO}_{2}$ Reactions

\begin{tabular}{crrrrr}
\hline \multirow{2}{*}{ Equation } & \multicolumn{5}{c}{$\Delta \mathrm{G}_{x}\left(\mathrm{cal} / \mathrm{mole} \mathrm{PuO}_{2}\right)$} \\
\cline { 2 - 6 } & $600^{\circ} \mathrm{C}$ & \multicolumn{1}{c}{$800^{\circ} \mathrm{C}$} & $1000^{\circ} \mathrm{C}$ & $1200^{\circ} \mathrm{C}$ & $1300^{\circ} \mathrm{C}$ \\
\hline 2 & 20,410 & 20,450 & 20,400 & 20,270 & 20,190 \\
4 & 6,370 & 6,840 & 7,260 & 7,630 & 7,800 \\
6 & 16,560 & 16,600 & 16,570 & 16,450 & 16,380 \\
8 & 16,320 & 16,460 & 16,520 & 16,520 & 16,510 \\
10 & 16,040 & 16,200 & 16,230 & 16,180 & 16,070 \\
12 & 22,910 & 23,570 & 24,320 & 24,820 & 25,120 \\
14 & 4,930 & 5,480 & 5,990 & 6,450 & 6,760 \\
16 & 2,350 & 2,890 & 3,330 & 3,620 & 3,820 \\
18 & 18,110 & 16,580 & 14,980 & 13,360 & 12,520 \\
20 & -800 & -140 & 450 & 990 & 1,240 \\
22 & $-27,130$ & $-24,520$ & $-21,990$ & $-19,560$ & $-18,230$ \\
24 & 26,270 & 26,390 & 24,470 & 23,500 & 22,990 \\
\hline
\end{tabular}

Reactions With $W-26 \%$ Re - The calculated partial molar free energies of mixing for the two constituents in $W-26 \%$ Re $(74.24$ atom $\% W$ and 25.76 atom \% Re) are given in Table 7 for the temperature range from 600 to $1400^{\circ} \mathrm{C}$.

Table 7. Partial Molar Free Energies of Mixing of the Components of $W-26 \% \operatorname{Re}$ Alloy

\begin{tabular}{ccc}
\hline $\begin{array}{c}\text { Temperature } \\
\left({ }^{\circ} \mathrm{C}\right)\end{array}$ & \multicolumn{3}{c}{$-\Delta \overline{\mathrm{G}}_{\mathrm{m}}(\mathrm{ca} / \mathrm{mole})$} \\
\cline { 2 - 3 } 6 & $\mathrm{~W}$ & $\mathrm{Re}$ \\
\hline 600 & 520 & 2350 \\
800 & 640 & 2890 \\
1000 & 750 & 3430 \\
1200 & 840 & 3970 \\
1300 & 930 & 4240 \\
1400 & 990 & 4510 \\
\hline
\end{tabular}

The free energies of formation for the oxides considered in the ${ }^{244} \mathrm{Cm}_{2} \mathrm{O}_{3}$ Fuel/W-26\% Re alloy reaction calculations are given in Table 8 . 
Table 8. Gibbs Free Energy of Formation of Oxides Involved in $W-26 \%$ Re Alloy/Curium Fuel Reactions

\begin{tabular}{crrrr}
\hline \multirow{2}{*}{$\begin{array}{c}\text { Temperature } \\
\left({ }^{\circ} \mathrm{C}\right)\end{array}$} & \multicolumn{4}{c}{$\Delta \mathrm{G}_{\mathrm{f}}(\mathrm{kcal} / \mathrm{mole})$} \\
\cline { 2 - 5 } & \multicolumn{1}{c}{$\mathrm{WO}_{2}$} & \multicolumn{1}{c}{$\mathrm{ReO}_{2}$} & $\mathrm{Pu}_{2} \mathrm{O}_{3}$ & $\mathrm{PuO}_{2}$ \\
\hline 600 & 101.16 & 66.80 & 352.12 & 215.55 \\
800 & 93.85 & 58.83 & 339.32 & 207.05 \\
1000 & 86.78 & 51.10 & 326.94 & 198.70 \\
1200 & 79.95 & 43.60 & 314.98 & 190.53 \\
1300 & 76.80 & 40.34 & 309.15 & 186.50 \\
1400 & 74.28 & 37.78 & 303.41 & 182.52
\end{tabular}

The values from Tables 7 and 8 were used to calculate the free energies for Eqg. 25-28 (shown below) over the $600-1400^{\circ} \mathrm{C}$ temperature range. Results of these calculations are given in Table 9.

$$
\begin{aligned}
& 3 W\left(\text { in W-26\% Re alloy) }(s)+2 \mathrm{Cm}_{2} \mathrm{O}_{3}(\mathrm{~s}) \rightarrow 3 \mathrm{WO}_{2}(\mathrm{~s})+4 \mathrm{Cm}(\mathrm{s})\right. \\
& W\left(\text { in } W-26 \% \operatorname{Re} \text { alloy) }(\mathrm{s})+4 \mathrm{PuO}_{2}(\mathrm{~s}) \rightarrow \mathrm{WO}_{2}(\mathrm{~s})+2 \mathrm{Pu}_{2} \mathrm{O}_{3}(\mathrm{~s})\right. \\
& 3 \operatorname{Re}\left(\text { in W-26\% } \operatorname{Re} \text { alloy) }(\mathrm{s})+2 \mathrm{Cm}_{2} \mathrm{O}_{3}(\mathrm{~s}) \rightarrow 3 \mathrm{ReO}_{2}(\mathrm{~s})+4 \mathrm{Cm}(\mathrm{s})\right. \\
& \operatorname{Re}\left(\text { in } W-26 \% \operatorname{Re} \text { alloy) }(\mathrm{s})+4 \mathrm{PuO}_{2}(\mathrm{~s}) \rightarrow \mathrm{ReO}_{2}(\mathrm{~s})+2 \mathrm{Pu}_{2} \mathrm{O}_{3}(\mathrm{~s})\right.
\end{aligned}
$$

Table 9. Gibbs Free Energy of Reaction for Eqs. 25-28

\begin{tabular}{ccccc}
\hline $\begin{array}{c}\text { Temperature } \\
\left({ }^{\circ} \mathrm{C}\right)\end{array}$ & \multicolumn{4}{c}{$\Delta \mathrm{G}$ (cal/mole of $\mathrm{Cm}_{2} \mathrm{O}_{3}$ or $\left.\mathrm{PuO}_{2}\right)$} \\
\cline { 2 - 5 } $\mathrm{Eq} \cdot 25$ & $\mathrm{Eq} \cdot 26$ & $\mathrm{Eq} \cdot 27$ & $\mathrm{Eq} \cdot 28$ \\
600 & 201,150 & 14,320 & 255,450 & 23,370 \\
800 & 199,500 & 14,080 & 255,420 & 23,400 \\
1000 & 197,900 & 13,730 & 255,440 & 23,320 \\
1200 & 196,300 & 13,260 & 255,540 & 23,130 \\
1300 & 195,350 & 12,960 & 255,000 & 22,900 \\
1400 & 193,480 & 12,490 & 253,520 & 22,490
\end{tabular}

Since the free energies are all positive for Eqs. 25-28, no reaction is predicted between W-26\% Re alloy and curium oxide sources between 600 and $1400^{\circ} \mathrm{C}$. 
Reactjons With Mo-46\% Re (69.50 atom \% Mo, 30.50 atom \% Re) - The partia1 molar free energy of mixing values for molybdenum and rhenium from 600 to $1400^{\circ} \mathrm{C}$ are shown in Table 10 ; the free energies of formation of $\mathrm{MoO}_{2}$, $\mathrm{ReO}_{2}, \mathrm{Pu}_{2} \mathrm{O}_{3}$, and $\mathrm{PuO}_{2}$ are shown in Table 11 .

Table 10. Partial Molar Free Energies of Mixing of the Components of Mo-46\% Re Alloy

\begin{tabular}{ccc}
\hline $\begin{array}{c}\text { Temperature } \\
\left({ }^{\circ} \mathrm{C}\right)\end{array}$ & \multicolumn{1}{c}{$-\Delta \bar{G}$ (cal/mole $)$} \\
\cline { 2 - 3 } 600 & Molybdenum & Rhenium \\
800 & 450 & 1620 \\
1000 & 590 & 2090 \\
1200 & 740 & 2570 \\
1300 & 880 & 3040 \\
1400 & 960 & 3270 \\
& 1030 & 3510 \\
\hline
\end{tabular}

Table 11. Gibbs Free Energies of Formation of $\mathrm{MoO}_{2}, \mathrm{ReO}_{2}, \mathrm{Pu}_{2} \mathrm{O}_{3}$, and $\mathrm{PuO}_{2}$

\begin{tabular}{ccccc}
\hline $\begin{array}{c}\text { Temperature } \\
\left({ }^{\circ} \mathrm{C}\right)\end{array}$ & \multicolumn{4}{c}{$-\Delta \mathrm{G}_{\mathrm{f}}(\mathrm{kcal} / \mathrm{mole})$} \\
\cline { 2 - 5 } $\mathrm{MoO}_{2}$ & $\mathrm{ReO}_{2}$ & $\mathrm{Pu}_{2} \mathrm{O}_{3}$ & $\mathrm{PuO}_{2}$ \\
\hline 600 & 95.60 & 66.80 & 352.12 & 215.55 \\
800 & 87.91 & 58.83 & 339.32 & 207.05 \\
1000 & 80.36 & 51.10 & 326.94 & 198.70 \\
1200 & 72.94 & 43.60 & 314.98 & 190.53 \\
1300 & 69.27 & 40.34 & 309.15 & 186.50 \\
1400 & 65.64 & 37.78 & 303.41 & 182.52 \\
\hline
\end{tabular}

The free energies from Tables 10 and 11 were used to calculate the free energies for Eqs. $29-32$ over the $600-1400^{\circ} \mathrm{C}$ temperature range. Results are shown in Table 12 .

$$
\begin{aligned}
& 3 \mathrm{Mo}\left(\text { in Mo- } 46 \% \text { Re alloy) (s) }+2 \mathrm{Cm}_{2} \mathrm{O}_{3}(\mathrm{~s}) \rightarrow 3 \mathrm{MoO}_{2}(\mathrm{~s})+4 \mathrm{Cm}(\mathrm{s})\right. \\
& \mathrm{Mo}\left(\text { in } \mathrm{Mo}-46 \% \operatorname{Re} \text { a11oy) (s) }+4 \mathrm{PuO}_{2}(\mathrm{~s}) \rightarrow \mathrm{MoO}_{2}(\mathrm{~s})+2 \mathrm{Pu}_{2} \mathrm{O}_{3}(\mathrm{~s})\right. \\
& 3 \operatorname{Re}\left(\text { in } \mathrm{Mo}-46 \% \operatorname{Re} \text { alloy) (s) }+2 \mathrm{Cm}_{2} \mathrm{O}_{3}(\mathrm{~s}) \rightarrow 3 \mathrm{ReO}_{2}(\mathrm{~s})+4 \mathrm{Cm}(\mathrm{s})\right. \\
& \operatorname{Re}\left(\text { in } \mathrm{Mo}-46 \% \operatorname{Re} \text { alloy) (s) }+4 \mathrm{PuO}_{2}(\mathrm{~s}) \rightarrow \mathrm{ReO}_{2}(\mathrm{~s})+2 \mathrm{Pu}_{2} \mathrm{O}_{3}(\mathrm{~s})\right.
\end{aligned}
$$

Since the free energies are all positive for Eqs. 29-32, no reaction is predicted between Mo- $46 \%$ Re alloy and the $\mathrm{Cm}_{2} \mathrm{O}_{3}$ product between 600 and $1400^{\circ} \mathrm{C}$. 
Table 12. Gibbs Free Energy of Reaction for Eqs. 29-32

\begin{tabular}{|c|c|c|c|c|}
\hline \multirow{2}{*}{$\begin{array}{c}\text { Temperature } \\
\left({ }^{\circ} \mathrm{C}\right)\end{array}$} & \multicolumn{4}{|c|}{$\Delta \mathrm{G}_{r}\left(\mathrm{cal} / \mathrm{mole}\right.$ of $\mathrm{Cm}_{2} \mathrm{O}_{3}$ or $\left.\mathrm{PuO}_{2}\right)$} \\
\hline & Eq. 29 & $\mathrm{Eq} \cdot 30$ & $\mathrm{Eq} \cdot 31$ & $\mathrm{Eq} \cdot 32$ \\
\hline 600 & 209,400 & 15,700 & 254,350 & 23,190 \\
\hline 800 & 208,340 & 15,660 & 254,220 & 23,200 \\
\hline 1000 & 207,510 & 15,330 & 254,140 & 23,100 \\
\hline 1200 & 206,900 & 15,030 & 254,130 & 22,900 \\
\hline 1300 & 206,680 & 14,850 & 253,540 & 22,660 \\
\hline 1400 & 206,500 & 14,660 & 252,010 & 22,240 \\
\hline
\end{tabular}

Reactions With Haynes 25 - The partial molar free energies of mixing for the constituents in Haynes 25 alloy are given in Table 13. The composition of Haynes 25 in atom \% is Co, 52.31; Ni, 10.64; Si, 2.22; Mn, 1.71; $\mathrm{C}, 0.52 ; \mathrm{P}, 0.06$; and $\mathrm{S}, 0.059$. The calculated free energies of formation of the various oxides are shown in Table 14.

Table 13. Partial Molar Free Energies of Mixing of the Constituents in Haynes 25 Alloy

\begin{tabular}{lrrrrr}
\hline \multirow{2}{*}{ Components } & \multicolumn{5}{c}{$-\Delta \mathrm{G}_{\mathrm{m}}(\mathrm{ca} 1 / \mathrm{mole})$} \\
\cline { 2 - 6 } & $600^{\circ} \mathrm{C}$ & $800^{\circ} \mathrm{C}$ & $1000^{\circ} \mathrm{C}$ & $1200^{\circ} \mathrm{C}$ & $1300^{\circ} \mathrm{C}$ \\
\hline Co & 1,060 & 1,320 & 1,580 & 1,830 & 1,960 \\
$\mathrm{Ni}$ & 3,880 & 4,780 & 5,670 & 6,560 & 7,000 \\
$\mathrm{Cr}$ & 2,200 & 2,760 & 3,330 & 3,890 & 4,180 \\
$\mathrm{~W}$ & 4,860 & 6,050 & 7,230 & 8,410 & 9,000 \\
$\mathrm{Fe}$ & 5,890 & 7,240 & 8,590 & 9,940 & 10,610 \\
$\mathrm{C}$ & 9,040 & 11,130 & 13,220 & 15,310 & 16,350 \\
$\mathrm{Si}$ & 6,330 & 7,840 & 9,360 & 10,870 & 11,630 \\
$\mathrm{Mn}$ & 6,980 & 8,600 & 10,220 & 11,830 & 12,640 \\
$\mathrm{P}$ & 12,840 & 15,790 & 18,730 & 21,680 & 23,150 \\
$\mathrm{~S}$ & 12,880 & 15,840 & 18,800 & 21,750 & 23,230 \\
& & & & & \\
\hline
\end{tabular}

The free energies from Tables 13 and 14 were used to calculate the free energies for Eqs. 33-52. Results are shown in Tables 15 and 16.

$$
\begin{aligned}
& 3 \mathrm{Co}\left(\text { in Haynes 25) (s) }+\mathrm{Cm}_{2} \mathrm{O}_{3}(\mathrm{~s}) \rightarrow 3 \mathrm{CoO}(\mathrm{s})+2 \mathrm{Cm}(\mathrm{s})\right. \\
& \mathrm{Co}\left(\text { In Haynes 25) (s) }+2 \mathrm{PuO}_{2}(\mathrm{~s}) \rightarrow \mathrm{CoO}(\mathrm{s})+\mathrm{Pu}_{2} \mathrm{O}_{3}(\mathrm{~s})\right. \\
& 3 \mathrm{Ni}\left(\text { in Haynes 25) }(\mathrm{s})+\mathrm{Cm}_{2} \mathrm{O}_{3}(\mathrm{~s}) \rightarrow 3 \mathrm{NiO}(\mathrm{s})+2 \mathrm{Cm}(\mathrm{s})\right. \\
& \mathrm{Ni}\left(\text { in Haynes 25) (s) }+2 \mathrm{PuO}_{2}(\mathrm{~s}) \rightarrow \mathrm{NiO}(\mathrm{s})+\mathrm{Pu}_{2} \mathrm{O}_{3}(\mathrm{~s})\right.
\end{aligned}
$$


Table 14. Gibbs Free Energy of Formation of Oxides Involved in Haynes 25/Curium Fuel Reactions

\begin{tabular}{lrrrrr}
\hline \multirow{2}{*}{ Constituents } & \multicolumn{5}{c}{$-\Delta \mathrm{G}_{\mathrm{f}}(\mathrm{kcal} / \mathrm{mole})$} \\
\cline { 2 - 6 } & $600^{\circ} \mathrm{C}$ & \multicolumn{1}{c}{$800^{\circ} \mathrm{C}$} & \multicolumn{1}{c}{$1000^{\circ} \mathrm{C}$} & $1200^{\circ} \mathrm{C}$ & $1300^{\circ} \mathrm{C}$ \\
\hline $\mathrm{CoO}$ & 39.46 & 35.37 & 31.02 & 27.08 & 24.96 \\
$\mathrm{NiO}$ & 39.06 & 35.01 & 31.01 & 27.10 & 25.15 \\
$\mathrm{Cr}_{2} \mathrm{O}_{3}$ & 203.92 & 189.81 & 175.72 & 161.67 & 154.63 \\
$\mathrm{WO}_{2}$ & 101.16 & 93.85 & 86.78 & 79.96 & 76.80 \\
$\mathrm{Fe}_{3} \mathrm{O}_{4}$ & 199.79 & 185.20 & 170.88 & 156.61 & 149.47 \\
$\mathrm{CO}_{2}$ & 94.55 & 94.36 & 94.24 & 94.02 & 94.00 \\
$\mathrm{SiO}_{2}$ & 172.92 & 164.60 & 156.33 & 148.10 & 144.00 \\
$\mathrm{MnO}$ & 76.82 & 73.30 & 69.76 & 66.23 & 74.27 \\
$\mathrm{P}_{2} \mathrm{O}_{3}$ & 212.19 & 200.88 & 189.69 & 178.62 & 173.16 \\
$\mathrm{SO}_{2}$ & 65.74 & 63.80 & 61.86 & 59.93 & 58.97 \\
$\mathrm{Pu}_{2} \mathrm{O}_{3}$ & 352.12 & 339.32 & 326.94 & 314.98 & 309.15 \\
$\mathrm{PuO}_{2}$ & 215.55 & 207.05 & 198.70 & 190.53 & 186.50 \\
& & & & &
\end{tabular}

$$
\begin{aligned}
& 2 \mathrm{Cr} \text { (in Haynes 25) (s) }+\mathrm{Cm}_{2} \mathrm{O}_{3}(\mathrm{~s}) \rightarrow \mathrm{Cr}_{2} \mathrm{O}_{3}(\mathrm{~s})+2 \mathrm{Cm}(\mathrm{s}) \\
& 2 \mathrm{Cr} \text { (in Haynes 25) (s) }+6 \mathrm{PuO}_{2}(\mathrm{~s}) \rightarrow \mathrm{Cr}_{2} \mathrm{O}_{3}(\mathrm{~s})+3 \mathrm{Pu}_{2} \mathrm{O}_{3}(\mathrm{~s}) \\
& 3 \mathrm{~W}\left(\text { in Haynes 25) }(\mathrm{s})+2 \mathrm{Cm}_{2} \mathrm{O}_{3}(\mathrm{~s}) \rightarrow 3 \mathrm{WO}_{2}(\mathrm{~s})+4 \mathrm{Cm}(\mathrm{s})\right. \\
& W(\text { in Haynes } 25)(\mathrm{s})+4 \mathrm{PuO}_{2}(\mathrm{~s}) \rightarrow \mathrm{WO}_{2}(\mathrm{~s})+2 \mathrm{Pu}_{2} \mathrm{O}_{3}(\mathrm{~s}) \\
& 9 \mathrm{Fe} \text { (in Haynes 25) (s) }+4 \mathrm{Cm}_{2} \mathrm{O}_{3}(\mathrm{~s}) \rightarrow 3 \mathrm{Fe}_{3} \mathrm{O}_{4}(\mathrm{~s})+8 \mathrm{Cm}(\mathrm{s}) \\
& 3 \mathrm{Fe} \text { (in Haynes 25) (s) }+8 \mathrm{PuO}_{2}(\mathrm{~s}) \rightarrow \mathrm{Fe}_{3} \mathrm{O}_{4}(\mathrm{~s})+4 \mathrm{Pu}_{2} \mathrm{O}_{3}(\mathrm{~s}) \\
& 3 \mathrm{C}\left(\text { in Haynes 25) }(\mathrm{s})+2 \mathrm{Cm}_{2} \mathrm{O}_{3}(\mathrm{~s}) \rightarrow 3 \mathrm{CO}_{2}(\mathrm{~g})+4 \mathrm{Cm}(\mathrm{s})\right. \\
& \mathrm{C}\left(\text { in Haynes 25) (s) }+4 \mathrm{PuO}_{2}(\mathrm{~s}) \rightarrow \mathrm{CO}_{2}(\mathrm{~g})+2 \mathrm{Pu}_{2} \mathrm{O}_{3}(\mathrm{~s})\right. \\
& 3 \mathrm{Si} \text { (in Haynes 25) (s) }+2 \mathrm{Cm}_{2} \mathrm{O}_{3}(\mathrm{~s}) \rightarrow 3 \mathrm{SiO}_{2}(\mathrm{~s})+4 \mathrm{Cm}(\mathrm{s})
\end{aligned}
$$

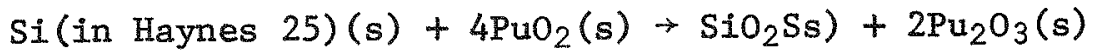

$$
\begin{aligned}
& 3 \mathrm{Mn} \text { (in Haynes 25) (s) }+\mathrm{Cm}_{2} \mathrm{O}_{3}(\mathrm{~s}) \rightarrow 3 \mathrm{MnO}(\mathrm{s})+2 \mathrm{Cm}(\mathrm{s}) \\
& \mathrm{Mn} \text { (in Haynes 25) (s) }+2 \mathrm{PuO}_{2}(\mathrm{~s}) \rightarrow \mathrm{MnO}(\mathrm{s})+\mathrm{Pu}_{2} \mathrm{O}_{3}(\mathrm{~s}) \\
& 2 \mathrm{P} \text { (in Haynes 25) (s) }+\mathrm{Cm}_{2} \mathrm{O}_{3}(\mathrm{~s}) \rightarrow \mathrm{P}_{2} \mathrm{O}_{3}(\mathrm{~s})+2 \mathrm{Cm}(\mathrm{s}) \\
& 2 \mathrm{P}\left(\text { in Haynes 25) (s) }+6 \mathrm{PuO}_{2}(\mathrm{~s}) \rightarrow \mathrm{P}_{2} \mathrm{O}_{3}(\mathrm{~s})+3 \mathrm{Pu}_{2} \mathrm{O}_{3}(\mathrm{~s})\right. \\
& 3 \mathrm{~S} \text { (in Haynes 25) (s) }+2 \mathrm{Cm}_{2} \mathrm{O}_{3}(\mathrm{~s}) \rightarrow 3 \mathrm{SO}_{2}(\mathrm{~g})+4 \mathrm{Cm}(\mathrm{s}) \\
& \mathrm{S}\left(\text { in Haynes 25) (s) }+4 \mathrm{PuO}_{2}(\mathrm{~s}) \rightarrow \mathrm{SO}_{2}(\mathrm{~g})+2 \mathrm{Pu}_{2} \mathrm{O}_{3}(\mathrm{~s})\right.
\end{aligned}
$$


Table 15. Free Energies of Reaction for Haynes $25 / \mathrm{Cm}_{2} \mathrm{O}_{3}$ Reactions

\begin{tabular}{|c|c|c|c|c|c|}
\hline \multirow[b]{2}{*}{ Equation } & \multicolumn{5}{|c|}{$\Delta \mathrm{G}_{\mathrm{x}}\left(\mathrm{cal} / \mathrm{mole} \mathrm{Cm}_{2} \mathrm{O}_{3}\right)$} \\
\hline & $600^{\circ} \mathrm{C}$ & $800^{\circ} \mathrm{C}$ & $1000^{\circ} \mathrm{C}$ & $1200^{\circ} \mathrm{C}$ & $1300^{\circ} \mathrm{C}$ \\
\hline 33 & 236,940 & 237,160 & 238,600 & 239,930 & 240,150 \\
\hline 35 & 246,580 & 248,620 & 250,890 & 253,360 & 254,710 \\
\hline 37 & 152,590 & 155,030 & 157,870 & 161,100 & 162,870 \\
\hline 39 & 207,680 & 207,620 & 207,620 & 207,660 & 207,460 \\
\hline 41 & 215,520 & 216,710 & 218,100 & 219,880 & 220,920 \\
\hline 43 & 223,850 & 214,480 & 205,420 & 196,910 & 192,690 \\
\hline 45 & 102,230 & 104,190 & 106,490 & 109,130 & 109,580 \\
\hline 47 & 142,600 & 145,210 & 148,310 & 151,790 & 154,290 \\
\hline 49 & $-46,580$ & $-30,850$ & $-14,970$ & 1,110 & 9,040 \\
\hline 51 & 272,820 & 267,370 & 262,340 & 257,710 & 255,540 \\
\hline
\end{tabular}

Table 16. Free Energies of Reaction for Haynes $25 / \mathrm{PuO}_{2}$ Reactions

\begin{tabular}{crrrrr}
\hline \multirow{2}{*}{ Equation } & \multicolumn{5}{c}{$\Delta G_{\mathrm{r}}\left(\mathrm{cal} / \mathrm{mole} \mathrm{PuO}_{2}\right)$} \\
\cline { 2 - 6 } & \multicolumn{1}{c}{$800^{\circ} \mathrm{C}$} & \multicolumn{1}{c}{$800^{\circ} \mathrm{C}$} & $1000^{\circ} \mathrm{C}$ & \multicolumn{1}{c}{$1200^{\circ} \mathrm{C}$} & $1300^{\circ} \mathrm{C}$ \\
34 & 20,290 & 20,360 & 20,510 & 20,420 & 20,430 \\
36 & 21,900 & 22,270 & 22,560 & 22,770 & 22,850 \\
38 & 6,230 & 6,670 & 7,060 & 7,390 & 7,550 \\
40 & 15,410 & 15,430 & 15,350 & 15,150 & 14,980 \\
42 & 315,880 & 299,080 & 281,880 & 264,310 & 255,420 \\
44 & 18,110 & 16,580 & 14,980 & 13,360 & 12,520 \\
46 & $-2,160$ & $-1,800$ & $-1,510$ & $-1,390$ & $-1,170$ \\
48 & 4,570 & 5,030 & 5,460 & 5,840 & 6,120 \\
50 & $-26,970$ & $-24,310$ & $-21,750$ & $-19,270$ & $-18,070$ \\
52 & 26,270 & 25,390 & 24,470 & 23,490 & 22,990 \\
& & & & & \\
\hline
\end{tabular}

Since the free energy of Eq. 49 is negative over the $600-1175^{\circ} \mathrm{C}$ range, the reaction of $\mathrm{Cm}_{2} \mathrm{O}_{3}$ with phosphorus of the alloy would be predicted. Likewise the free energy of Eqs. 46 and 50 are negative and the reaction of $\mathrm{PuO}_{2}$ with the silicon and phosphorus of the alloy is predicted. Although it was assumed that no compound formation between the fuel and the constituents of the alloy would occur, phosphorus is so reactive that it is highly likely that it may be involved in a compound formation with one of the other constituents of the alloy, and this could change the thermodynamic picture. However, the small percentage of silicon is also expected to react. 
Reactions With $\mathrm{Pt}-2608 \mathrm{M}$ - The composition of $\mathrm{Pt}-2608 \mathrm{M}$ in atom percent is $\mathrm{Pt}, 52.27 ; \mathrm{Rh}, 39.33 ; \mathrm{W}, 6.77$; and $\mathrm{Ti}, 1.63$. The partial molar free energies of mixing for $\mathrm{Pt}, \mathrm{Rh}, \mathrm{W}$, and $\mathrm{Ti}$ were calculated; the results are shown in Table 17.

Table 17. Partial Molar Free Energies of Mixing of Components of $\mathrm{Pt}-26 \% \mathrm{Rh}-8 \% \mathrm{~W}-0.5 \% \mathrm{Ti}$

\begin{tabular}{ccccr}
\hline \multirow{2}{*}{$\begin{array}{c}\text { Temperature } \\
\left({ }^{\circ} \mathrm{C}\right)\end{array}$} & \multicolumn{4}{c}{$-\Delta \overline{\mathrm{G}}_{\mathrm{m}}$ (cal/mole) } \\
\cline { 2 - 5 } & $\mathrm{Pt}$ & $\mathrm{Rh}$ & $\mathrm{W}$ & $\mathrm{Ti}$ \\
\hline 600 & 1040 & 1570 & 4420 & 6,980 \\
800 & 1300 & 1940 & 5490 & 8,620 \\
1000 & 1560 & 2320 & 6560 & 10,260 \\
1200 & 1820 & 2690 & 7630 & 11,890 \\
1300 & 1950 & 2870 & 8160 & 12,710 \\
\hline
\end{tabular}

The calculated free energies of formation of $\mathrm{Pt}_{3} \mathrm{O}_{4}, \mathrm{Rh}_{2} \mathrm{O}$, $\mathrm{WO}_{2}, \mathrm{TiO}, \mathrm{Pu}_{2} \mathrm{O}_{3}$, and $\mathrm{PuO}_{2}$ are shown in Table 18. Platinum will not react with oxygen at $600-1300^{\circ} \mathrm{C}$, but rhodium, tungsten, and titanium will react with oxygen at $600-1300^{\circ} \mathrm{C}$.

Tab1e 18. Gibbs Free Energy of Formation of $\mathrm{Pt}_{3} \mathrm{O}_{4}, \mathrm{Rh}_{2} \mathrm{O}, \mathrm{WO}_{2}, \mathrm{TiO}, \mathrm{Pu}_{2} \mathrm{O}_{3}$, and $\mathrm{PuO}_{2}$

\begin{tabular}{ccccccc}
\hline $\begin{array}{c}\text { Temperature } \\
\left({ }^{\circ} \mathrm{C}\right)\end{array}$ & \multicolumn{7}{c}{$\Delta \mathrm{G}_{\mathrm{f}}(\mathrm{cal} / \mathrm{mole})$} \\
\cline { 2 - 7 } & $\mathrm{Pt}_{3} \mathrm{O}_{4}$ & $\mathrm{Rh}_{2} \mathrm{O}$ & $\mathrm{WO}_{2}$ & $\mathrm{TiO}$ & $\mathrm{Pu}_{2} \mathrm{O}_{3}$ & $\mathrm{PuO}_{2}$ \\
\hline & & & & & & \\
600 & 10,300 & $-11,350$ & $-101,160$ & $-103,780$ & $-352,120$ & $-215,550$ \\
800 & 26,400 & $-9,090$ & $-93,850$ & $-99,380$ & $-339,320$ & $-207,050$ \\
1000 & 42,080 & $-6,940$ & $-86,780$ & $-95,080$ & $-326,940$ & $-198,700$ \\
1200 & 57,330 & $-4,900$ & $-79,960$ & $-90,870$ & $-314,980$ & $-190,530$ \\
1300 & 64,790 & $-3,910$ & $-76,800$ & $-88,810$ & $-309,150$ & $-186,500$ \\
\hline
\end{tabular}

The free energies from Tables 17 and 18 were used to calculate the free energies for Eqs. 53-58. Results are shown in Table 19.

$$
\begin{aligned}
& 6 \mathrm{Rh} \text { (in Pt-2608M alloy) (s) }+\mathrm{Cm}_{2} \mathrm{O}_{3}(\mathrm{~s}) \rightarrow 3 \mathrm{Rh}_{2} \mathrm{O}(\mathrm{s})+2 \mathrm{Cm}(\mathrm{s}) \\
& 2 \mathrm{Rh}\left(\text { in Pt-2608M alloy) (s) }+2 \mathrm{PuO}_{2}(\mathrm{~s}) \rightarrow \mathrm{Rh}_{2} \mathrm{O}(\mathrm{s})+\mathrm{Pu}_{2} \mathrm{O}_{3}(\mathrm{~s})\right. \\
& 3 \mathrm{~W}\left(\text { in Pt-2608M alloy) }(\mathrm{s})+2 \mathrm{Cm}_{2} \mathrm{O}_{3}(\mathrm{~s}) \rightarrow 3 \mathrm{WO}_{2}(\mathrm{~s})+4 \mathrm{Cm}(\mathrm{s})\right. \\
& \mathrm{W}\left(\text { in Pt-2608M alloy) }(\mathrm{s})+4 \mathrm{PuO}_{2}(\mathrm{~s}) \rightarrow \mathrm{WO}_{2}(\mathrm{~s})+2 \mathrm{Pu}_{2} \mathrm{O}_{3}(\mathrm{~s})\right. \\
& 3 \mathrm{Ti}\left(\text { in Pt-2608M alloy) (s) }+\mathrm{Cm}_{2} \mathrm{O}_{3}(\mathrm{~s}) \rightarrow 3 \mathrm{TiO}(\mathrm{s})+2 \mathrm{Cm}(\mathrm{s})\right. \\
& \mathrm{Ti}\left(\text { in Pt-2608M alloy) (s) }+2 \mathrm{PuO}_{2}(\mathrm{~s}) \rightarrow \mathrm{TiO}(\mathrm{s})+\mathrm{Pu}_{2} \mathrm{O}_{3}(\mathrm{~s})\right.
\end{aligned}
$$


Table 19. Gibbs Free Energy of Reaction for Eqs. 53-58

\begin{tabular}{cccccccc}
\hline $\begin{array}{c}\text { Temperature } \\
\left({ }^{\circ} \mathrm{C}\right)\end{array}$ & \multicolumn{6}{c}{$\Delta \mathrm{G}_{\mathrm{r}}(\mathrm{kcal} / \mathrm{mole}$ of } & $\mathrm{Cm}_{2} \mathrm{O}_{3}$ or $\left.\mathrm{PuO}_{2}\right)$ \\
\hline & $\mathrm{Eq} \cdot 53$ & $\mathrm{Eq} \cdot 54$ & $\mathrm{Eq} \cdot 55$ & $\mathrm{Eq} \cdot 56$ & $\mathrm{Eq} \cdot 57$ & $\mathrm{Eq} \cdot 58$ \\
\hline 600 & 327.49 & 35.38 & 207.01 & 15.30 & 61.72 & -8.92 \\
800 & 323.69 & 34.78 & 206.78 & 15.30 & 67.04 & -8.00 \\
1000 & 320.04 & 34.09 & 206.61 & 15.18 & 72.48 & -7.18 \\
1200 & 316.42 & 33.28 & 206.49 & 14.96 & 78.04 & -6.45 \\
1300 & 315.07 & 32.85 & 206.19 & 14.77 & 80.85 & -6.12
\end{tabular}

Since the free energies are positive for Eqs. 53, 55, and 57, $\mathrm{Cm}_{2} \mathrm{O}_{3}$ is not expected to react with the alloy. However, the reaction of titanium with $\mathrm{PuO}_{2}$ has a negative free energy, hence the $\mathrm{PuO}_{2}$ of the curium product is expected to react with the titanium of the alloy.

In conclusion, it appears from thermodynamic considerations that the $\mathrm{Pt}-26 \% \mathrm{Rh}-8 \% \mathrm{~W}-0.5 \% \mathrm{Ti}$ alloy will react with the $\mathrm{Cm}_{2} \mathrm{O}_{3}$ fuel containing $5 \% \mathrm{PuO}_{2}$ at $600-1300^{\circ} \mathrm{C}$.

Silicon Nitride - Several possible reactions of $\mathrm{Si}_{3} \mathrm{~N}_{4}$ with the curium heat source product are conceivable. Some of the possible products are $\mathrm{CmN}, \mathrm{N}_{2}, \mathrm{SiO}_{2}, \mathrm{CmSi}, \mathrm{Cm}_{2} \mathrm{Si}_{3}$, and $\mathrm{CmSi}_{2}$. The thermodynamic data needed for the calculating of the free energies of reaction to produce curium silicides are not available. Hence, the free energy calculations were made on the $\mathrm{CmN}, \mathrm{N}_{2}$, and $\mathrm{SiO}_{2}$ only. Since the actinide silicides are probably among the weaker compounds, they may not be important anyway.

The free energies of formation of $\mathrm{Si}_{3} \mathrm{~N}_{4}, \mathrm{CeN}, \mathrm{SiO}_{2}, \mathrm{Pu}_{2} \mathrm{O}_{3}$, and $\mathrm{PuO}_{2}$ were calculated from a data calculation by 0 . Kubaschewski et $a Z_{0}, 4$ a compilation by Glassner, ${ }^{2}$ a survey by $\mathrm{K}$. A. Gschneidner, ${ }^{5}$ and a review article by Detting. ${ }^{3}$. The results are shown in Table 20 .

Table 20. Gibbs Free Energy of Formation of $\mathrm{Si}_{3} \mathrm{~N}_{4}, \mathrm{CeN}, \mathrm{SiO}_{2}, \mathrm{Pu}_{2} \mathrm{O}_{3}$, and $\mathrm{PuO}_{2}$

\begin{tabular}{clllll}
\hline \multirow{2}{*}{$\begin{array}{c}\text { Temperature } \\
\left({ }^{\circ} \mathrm{C}\right)\end{array}$} & \multicolumn{5}{c}{$-\Delta \mathrm{G}_{f}(\mathrm{kcal} / \mathrm{mole})$} \\
\cline { 2 - 6 } $\mathrm{Si}_{3} \mathrm{~N}_{4}$ & $\mathrm{CeN}$ & $\mathrm{SiO}_{2}$ & $\mathrm{Pu}_{2} \mathrm{O}_{3}$ & $\mathrm{PuO}_{2}$ \\
\hline 600 & 240.79 & 56.18 & 172.92 & 352.12 & 215.55 \\
800 & 260.99 & 51.18 & 164.60 & 339.32 & 207.05 \\
1000 & 282.05 & 46.18 & 156.33 & 326.94 & 198.70 \\
1200 & 303.97 & 41.18 & 148.10 & 314.98 & 190.53 \\
1300 & 315.14 & 38.68 & 144.00 & 309.15 & 186.50 \\
& & & & & \\
\hline
\end{tabular}


The data from Table 20 were used to calculate the free energies of Eqs. 59 and 60. The $\mathrm{CeN}$ was used as a stand-in for $\mathrm{CmN}$ and $\mathrm{Pu}_{2} \mathrm{O}_{3}$ was used as a stand-in for $\mathrm{Cm}_{2} \mathrm{O}_{3}$. Results are shown in Table 21 .

$$
\begin{aligned}
& 2 \mathrm{Cm}_{2} \mathrm{O}_{3}(\mathrm{~s})+\mathrm{Si}_{3} \mathrm{~N}_{4}(\mathrm{~s}) \rightarrow 4 \mathrm{CmN}(\mathrm{s})+3 \mathrm{SiO}_{2}(\mathrm{~s}) \\
& 12 \mathrm{PuO}_{2}(\mathrm{~s})+\mathrm{Si}_{3} \mathrm{~N}_{4}(\mathrm{~s}) \rightarrow 6 \mathrm{Pu}_{2} \mathrm{O}_{3}(\mathrm{~s})+3 \mathrm{SiO}_{2}(\mathrm{~s})+2 \mathrm{~N}_{2}(\mathrm{~g})
\end{aligned}
$$

Table 21. Gibbs Free Energy for Eqs. 59 and 60

\begin{tabular}{ccc}
\hline $\begin{array}{c}\text { Temperature } \\
\left({ }^{\circ} \mathrm{C}\right)\end{array}$ & $\Delta \mathrm{G}_{\mathrm{r}}\left(\mathrm{cal} / \mathrm{mole}\right.$ of $\mathrm{Cm}_{2} \mathrm{O}_{3}$ or $\left.\mathrm{PuO}_{2}\right)$ \\
\cline { 2 - 3 } 600 & $\mathrm{Eq} .59$ & $\mathrm{Eq} .60$ \\
800 & 156,960 & 16,320 \\
1000 & 171,740 & 17,980 \\
1200 & 187,300 & 19,660 \\
1300 & 203,640 & 21,350 \\
& 212,040 & 22,190 \\
\hline
\end{tabular}

Since the free energies of Eqs. 59 and 60 are positive, the $\mathrm{Si}_{3} \mathrm{~N}_{4}$ should not react with the curium oxide heat source product to produce $\mathrm{CmN}, \mathrm{N}_{2}$, or $\mathrm{SiO}_{2}$. Nothing definite can be said about the reactions of $\mathrm{Si}_{3} \mathrm{~N}_{4}$ with the curium oxide product to give a silicide but this is not expected since the silicides are probably weaker compounds than the nitrides. From the standpoint of thermodynamic calculations, the $\mathrm{Si}_{3} \mathrm{~N}_{4}$ should not react with the curium oxide product. Compatibility studies are needed to verify the conclusions.

\section{${ }^{244} \mathrm{Cm}_{2} \mathrm{O}_{3}$ Property Characterization}

Helium Release ( $P$. Angelini)

The three helium release experiments initiated in December 1973 were completed. Experimental procedures and initial data were reported previously.1 The plots of helium release rate versus time for samples A $\left(500^{\circ} \mathrm{C}\right)$, B $\left(800^{\circ} \mathrm{C}\right)$, and $\mathrm{C}\left(650^{\circ} \mathrm{C}\right)$ are presented in Fig. 1. During each incremental measuring time, the release rate is recorded and integrated such that the amount of helium emitted over that incremental time is obtained. The abscissa of Fig. 1 represents the time from initial heating of the sample to the end of the incremental measuring time for the release rate.

The experiments were continued uninterrupted up to time period $\mathrm{J}$ (see Fig. 1). At time $J$ the temperature of a specific sample was decreased, and the sample unloaded in an inert atmosphere glove box. The sample pellet was then photographed, and its dimensions and mass measured. It was then cracked with approximately one-half of the sample used for an $x$-ray diffraction study and the remainder reweighed and reloaded into the 


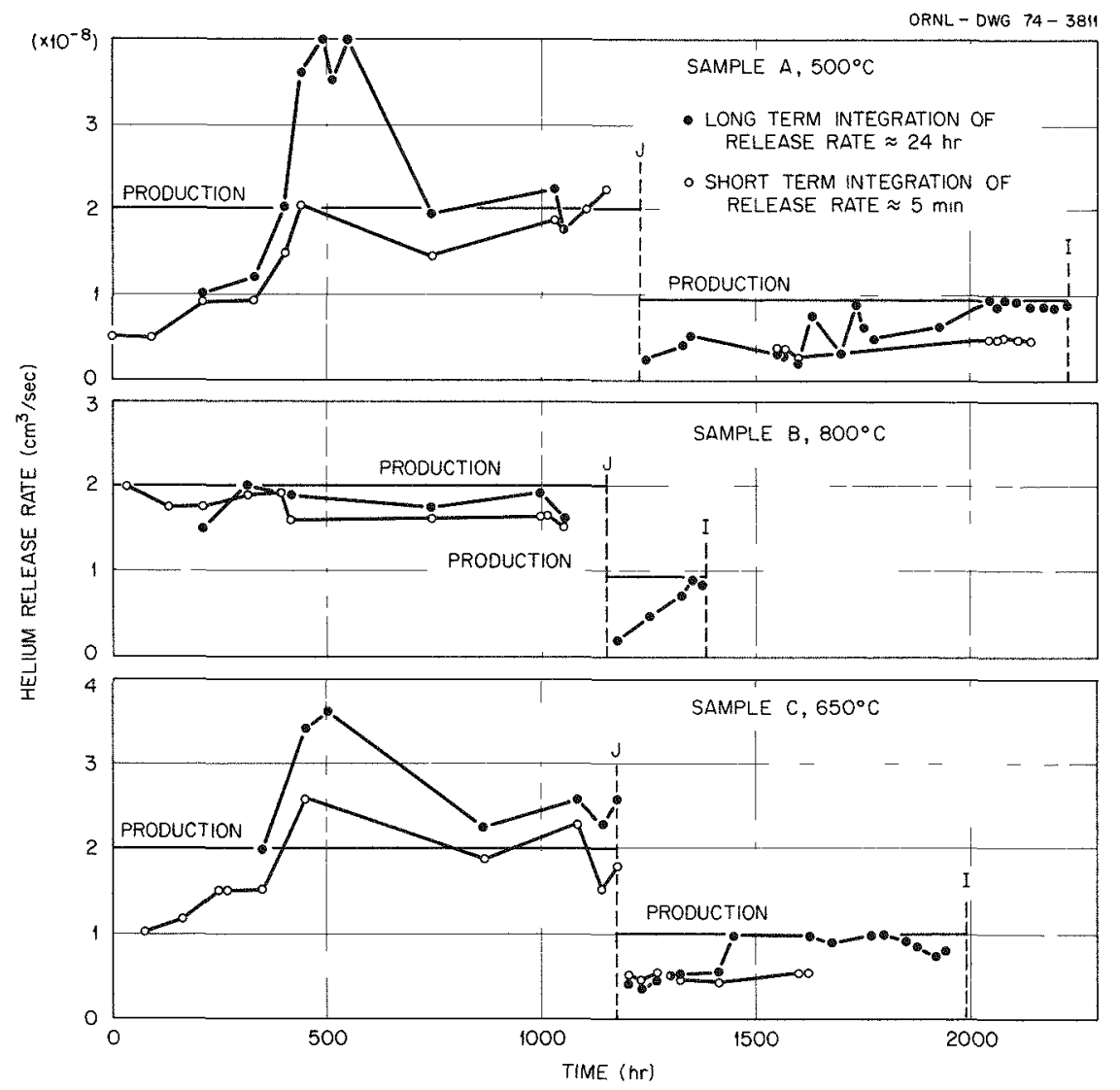

Fig. 1. Helium Release Rate vs Time for ${ }^{244} \mathrm{Cm}_{2} \mathrm{O}_{3}$ Samples.

furnace and reheated to the same previous temperature. The time period necessary to perform these operations was approximately six hours per sample. The $x$-ray diffraction scans were performed usually a day after the unloading procedure. The three samples were unloaded on different days in order to keep their exposure at ambient temperature to a minimum.

The mass and dimensional data for each sample measured at the beginning of the experiment (time 0 ) and at time $J$ are presented in Table 22.

Table 22. Mass and Dimensional Data

\begin{tabular}{ccccccccc}
\hline \multirow{2}{*}{ Sample } & $\begin{array}{c}\text { Temperature } \\
\left({ }^{\circ} \mathrm{C}\right)\end{array}$ & $\begin{array}{c}\text { Mass } \\
(\mathrm{g})\end{array}$ & $\begin{array}{c}\text { Height } \\
(\mathrm{mils})\end{array}$ & $\begin{array}{c}\text { Density } \\
\left(\mathrm{g} / \mathrm{cm}^{3}\right)\end{array}$ & $\begin{array}{c}\text { Mass } \\
(\mathrm{g})\end{array}$ & $\begin{array}{c}\text { Height } \\
(\mathrm{mils})\end{array}$ & $\begin{array}{c}\text { Density } \\
\left(\mathrm{g} / \mathrm{cm}^{3}\right)\end{array}$ \\
\hline $\mathrm{A}$ & 500 & 0.2278 & 52.3 & 10.44 & 0.228 & 52.2 & 10.47 \\
$\mathrm{C}$ & 650 & 0.2374 & 53.4 & 10.66 & 0.238 & 53.5 & 10.67 \\
$\mathrm{~B}$ & 800 & 0.2291 & 53.7 & 10.23 & 0.229 & 52.6 & 10.44 \\
\hline
\end{tabular}


The mass measurements are within experimental error. No increase or decrease in mass occurred in any of the samples during the more than $1100 \mathrm{hr}$ at temperature. If there was any oxidation or reduction of the samples during this time, a change in mass would have occurred and been measured. Thus, the samples did not change stoichiometry during the test. The measurement of height for pellets $A\left(500^{\circ} \mathrm{C}\right)$ and $\mathrm{C}\left(650^{\circ} \mathrm{C}\right)$ showed no differences at time $\mathrm{J}$. A significant height change occurred in sample $\mathrm{B}\left(800^{\circ} \mathrm{C}\right)$. The sample increased in density during the time period $\mathrm{J}$. This is a significant difference between the lower temperature experiments $(500$ and $650^{\circ} \mathrm{C}$ ) and the $800^{\circ} \mathrm{C}$ experiment and is an additional phenomenon which shows that a different mechanism for helium release in $\mathrm{Cm}_{2} \mathrm{O}_{3}$ occurs at temperatures lower than $650^{\circ} \mathrm{C}$.

Prior to unloading of the samples, it was thought that perhaps some macroscopic damage had occurred to sample pellets $A$ and $C$ due to their emission of a large fraction of their helium inventory during an earlier time in the experiment. In inspecting the sample pellets during the unloading period, no major macroscopic defects could be seen in any of the pellets. The pellets were photographed (Fig. 2), and no anomalous expansion occurred in any of the pellets. It can be seen from the photographs that there is a slight difference in surface appearance of the 500 and $650^{\circ} \mathrm{C}$ samples and the $800^{\circ} \mathrm{C}$ sample. The pellets were broken using a mortar and pestle. Sample A $\left(500^{\circ} \mathrm{C}\right)$ broke into many pieces and was the most easily broken of the three pellets. The $800^{\circ} \mathrm{C}$ pellet was the hardest to break. This would indicate that some macroscopic structural damage did occur to the lower temperature pellets $\mathrm{A}$ and $\mathrm{C}$.

$X$-ray diffraction scans made from 10 to $80^{\circ} 2 \theta$ showed that the lower the sample temperature the larger the monoclinic cell parameters and the broader the diffraction peaks. Also the diffraction peaks above $60^{\circ} 2 \theta$ were not observed for the 500 and $650^{\circ} \mathrm{C}$ samples. The pattern obtained from the $800^{\circ} \mathrm{C}$ sample indicated that the monoclinic cell parameters were larger than those obtained for a standard $\mathrm{Cm}_{2} \mathrm{O}_{3}$ monoclinic sample. X-ray diffraction peaks up to $80^{\circ} 2 \theta$ were measured for the $800^{\circ} \mathrm{C}$ sample. These results show that the crystallites in the 500 and $650^{\circ} \mathrm{C}$ samples were smaller than those of the $800^{\circ} \mathrm{C}$ sample. The absence of the high angle lines indicates additional damage to the crystal structure of the 500 and $650^{\circ} \mathrm{C}$ samples.

The evidence of the dimensional differences, the surface appearance, the mechanical strength of the pellets, and the differences observed in the $x$-ray diffraction scans show that the lower temperature pellets did undergo greater crystallite and structural damage than the $800^{\circ} \mathrm{C}$ pellet. The data reinforce the theory of a different helium release mechanism operating at the lower temperatures.

After the samples were weighed and reloaded into the furnaces, the helium release experiment continued until the helium release rate of the samples was equal to the helium generation rate. At a time labeled I on Fig. 1, the samples were transferred to the high-temperature vacuum furnace and degassed to a temperature of $1900^{\circ} \mathrm{C}$. The results of the helium inventory measurements are presented in Table 23. 


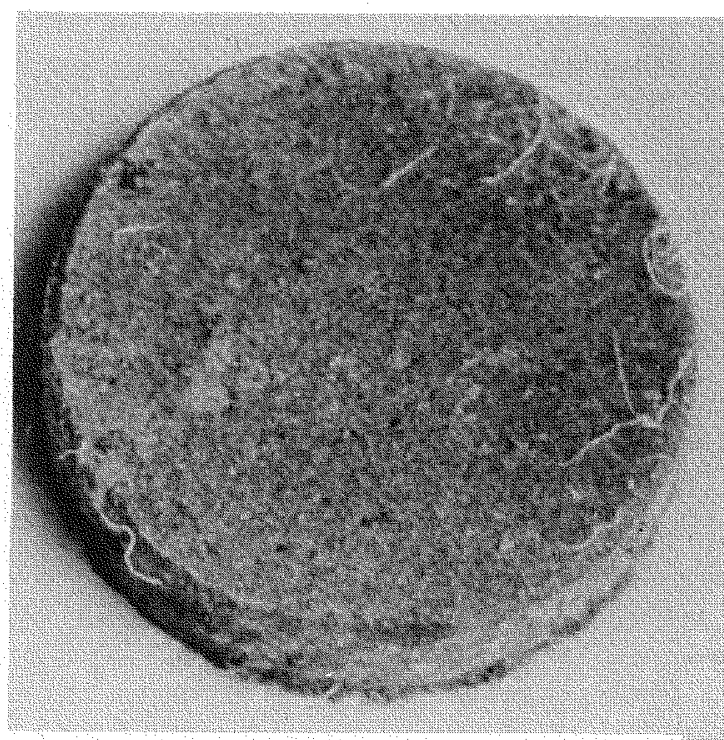

Sample A, $500^{\circ} \mathrm{C}, 1230 \mathrm{hr}$

Sample B, $800^{\circ} \mathrm{C}, 1150 \mathrm{hr}$

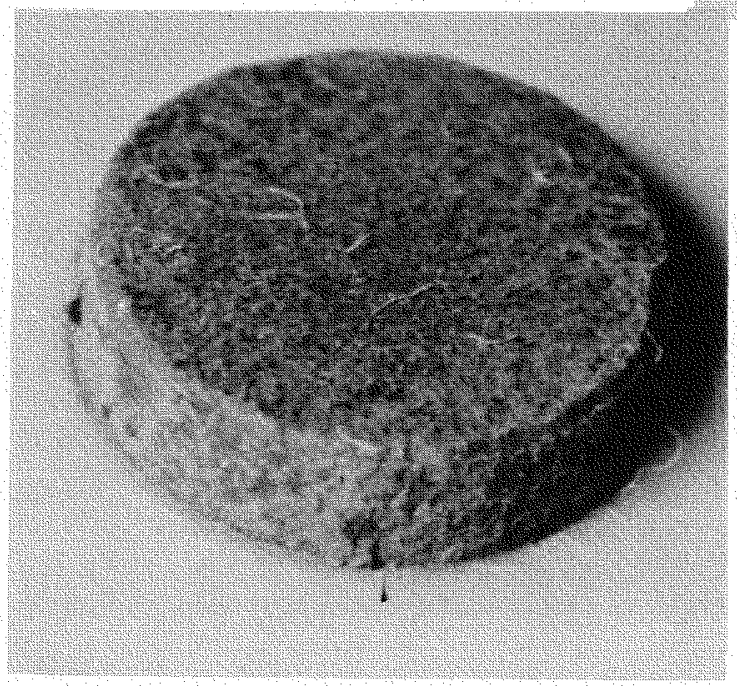

Sample C, $650^{\circ} \mathrm{C}, 1175 \mathrm{hr}$

Fig. 2. $\quad{ }^{44} \mathrm{Cm}_{2} \mathrm{O}_{3}$ Pellets After Helium Release Experiment. 
Table 23. Helium Inventory Measurements

\begin{tabular}{cccc}
\hline Sample & $\begin{array}{c}\text { Temperature } \\
\left({ }^{\circ} \mathrm{C}\right)\end{array}$ & $\begin{array}{c}\text { Helium Inventory } \\
\left(\mathrm{cm}^{3} \mathrm{He} / \mathrm{g} \mathrm{Cm} \mathrm{C}_{3}\right)\end{array}$ & $\begin{array}{c}\text { Diffusion Parameter } \mathrm{D}^{\prime} \\
(1 / \mathrm{sec})\end{array}$ \\
\hline $\mathrm{A}$ & 500 & 0.262 & $2.26 \times 10^{-8}$ \\
$\mathrm{C}$ & 650 & 0.194 & $3.04 \times 10^{-8}$ \\
B & 800 & 0.0582 & $1.07 \times 10^{-7}$ \\
\hline
\end{tabular}

The diffusion parameter data are plotted on Fig. 3 with the previous data

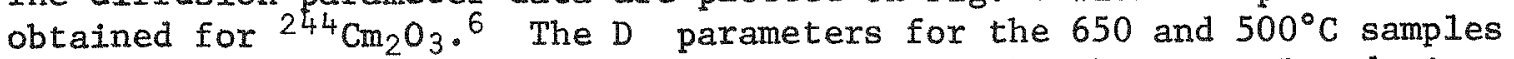
are near the extrapolated curve. On observing the three samples during this experiment, various nonsteady state situations could be seen in each. The diffusion parameter probably varied in absolute value during the course of the experiment for each sample but much more drastically for the 500 and $650^{\circ} \mathrm{C}$ samples. In observing the results in Fig. 1 , an

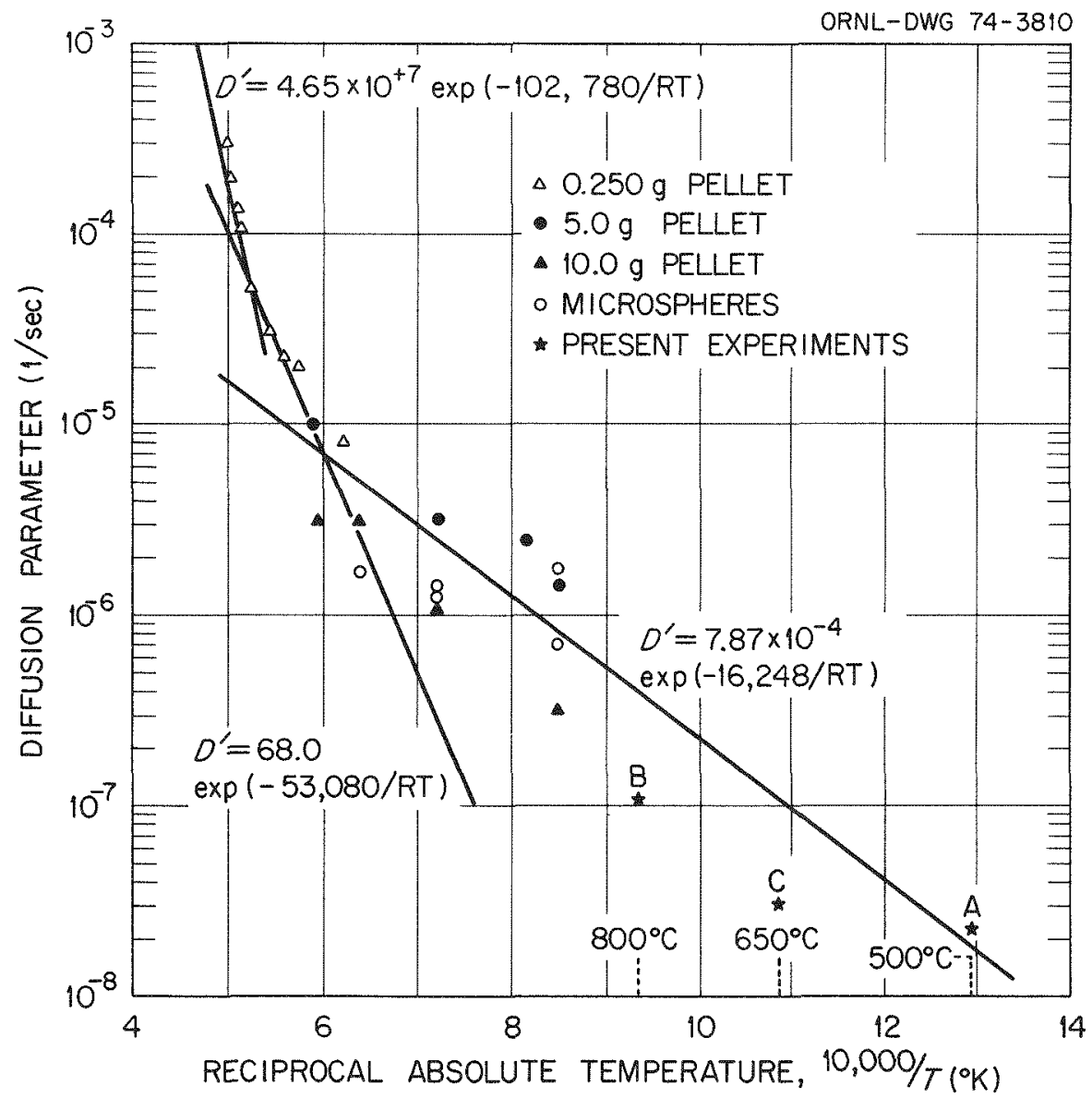

Fig. 3. Helium Diffusion Parameters for ${ }^{244} \mathrm{Cm}_{2} \mathrm{O}_{3}$. 
anomaly was noted in that after the reloading period the helium release rate for the three samples decreased from its previous steady-state value. As time progressed, the type of release changed for the $A\left(500^{\circ} \mathrm{C}\right)$ and $\mathrm{C}\left(650^{\circ} \mathrm{C}\right)$ samples. The burst-type helium release again became prominent during the remainder of the experiment for samples $A$ and $C$. The $A\left(500^{\circ} \mathrm{C}\right)$ and $\mathrm{C}\left(650^{\circ} \mathrm{C}\right)$ experiments showed that there is an additional helium release mechanism at these temperatures. Additional experiments would be necessary in this temperature range in order to better understand the phenomena occurring at these temperatures. At these temperatures there are many factors which could influence the helium release characteristics of samples, and these coupled with the long time period necessary for a steady-state condition to develop greatly increase the problem of clarifyIng the helium release phenomena near the $650^{\circ} \mathrm{C}$ temperature range.

Four isochronal helium release experiments were performed using the remainder of the ${ }^{244} \mathrm{Cm}_{2} \mathrm{O}_{3}$ fuel from helium release experiment $\mathrm{C}\left(650^{\circ} \mathrm{C}\right)$. These were performed to provide additional information as to the types of helium release mechanisms operating in the 500 and $800^{\circ} \mathrm{C}$ temperature range.

The experimental conditions of the four experiments are presented in Table 24.

Table 24. Experimental Conditions of the

Isochronal Anneal Experiments for ${ }^{244} \mathrm{Cm}_{2} \mathrm{O}_{3}$

\begin{tabular}{ccc}
\hline $\begin{array}{c}\text { Experiment } \\
\text { Number }\end{array}$ & $\begin{array}{c}\text { Initial Annealing } \\
\text { Temperature }\left({ }^{\circ} \mathrm{C}\right)\end{array}$ & $\begin{array}{c}\text { Time Period at Ambient } \\
\text { Temperature (days) }\end{array}$ \\
\hline 1 & 800 & 3 \\
2 & 800 & 20 \\
3 & 1100 & 20 \\
4 & 1100 & 4 \\
\hline
\end{tabular}

In these experiments the initial annealing temperature was held for approximately $1 \mathrm{hr}$. The sample was then cooled over a $3-\mathrm{hr}$ period to ambient temperature with the sample held at ambient temperature for the desired time period as shown in Table 24. The concentation of helium in the sample increased at ambient temperature during the desired time period. During each of the isochronal anneal experiments, the sample was maintained at the desired temperature for one half hour with the sample's temperature increased in $100^{\circ} \mathrm{C}$ intervals.

The data obtained during the experiments are presented in Fig. 4. In observing the data from experiment 1 , the onset of a definite change in helium release characteristics of the sample occurred near the $600^{\circ} \mathrm{C}$ temperature range. In experiment 2 the sample was maintained at ambient temperature for 20 days (approximately seven times longer than experiment 1). Again the onset of the measurable change of the helium release 


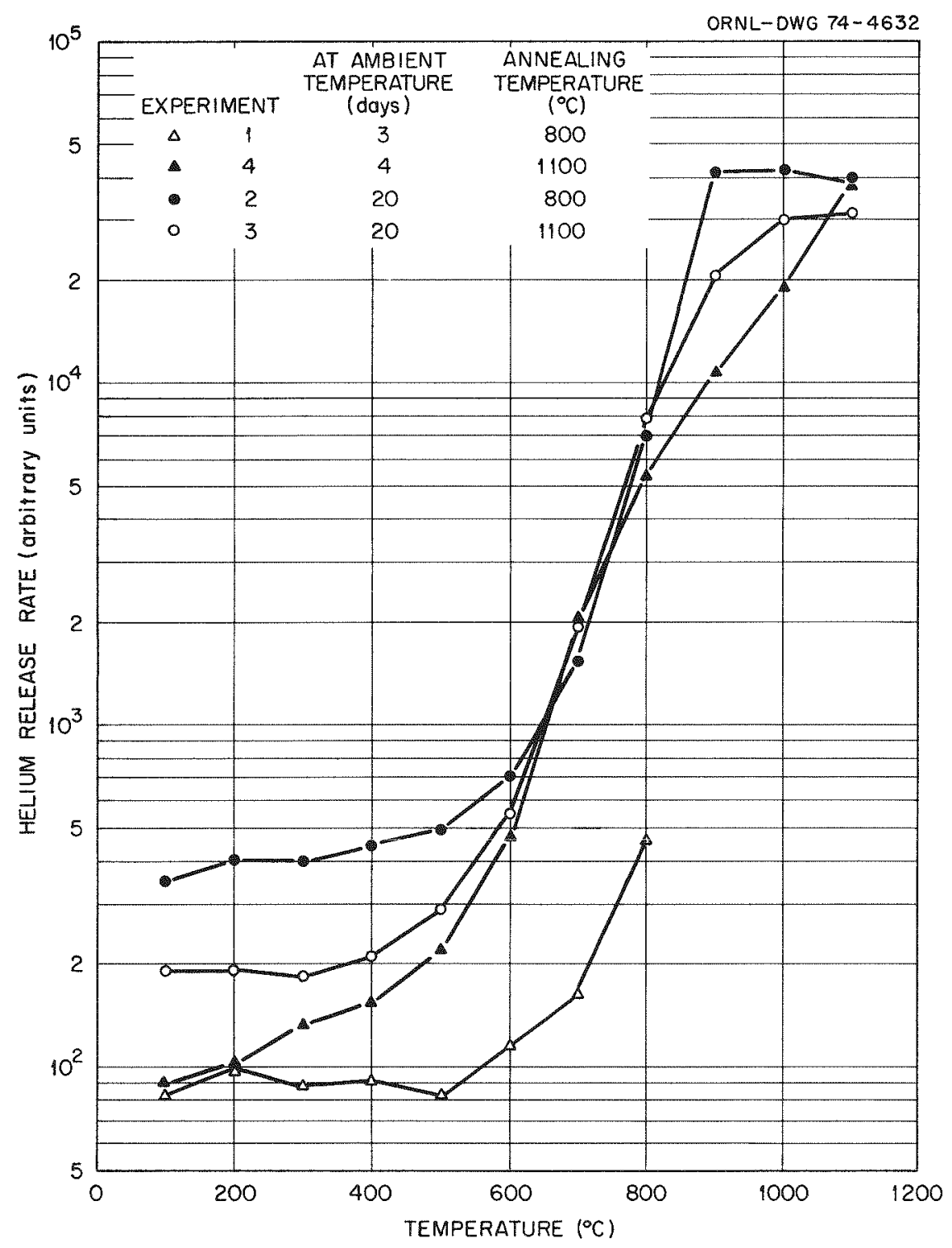

Fig. 4. Isochronal Anneal Experiments for ${ }^{244} \mathrm{Cm}_{2} \mathrm{O}_{3}$.

characteristics occurred near the $600^{\circ} \mathrm{C}$ temperature. Thus the initial helium concentration in the sample within the concentration range obtained in the present experiments does not significantly change the temperature at which there is a definite change in the helium release mechanism.

The experimental conditions were changed such that the initial annealing temperature was increased from 800 to $1100^{\circ} \mathrm{C}$. The data from experiment 3 (20-day period at ambient temperature) indicate that the onset of measurable change in the helium release characteristics again occurred near the $600^{\circ} \mathrm{C}$ temperature. In this experiment, burst-type helium release was observed to begin at $300^{\circ} \mathrm{C}$, to peak at $800^{\circ} \mathrm{C}$, and to continue to $1100^{\circ} \mathrm{C}$. In experiment 4 (4-day period at ambient temperature) the onset of the 
change in the helium release again occurred near $600^{\circ} \mathrm{C}$. In addition there was no burst-type helium release observed during the experiment. Thus changing the initial annealing temperature from 800 to $1100^{\circ} \mathrm{C}$ in the present experiments does not significantly change the temperature at which the onset of the helium release mechanism operative above the $600^{\circ} \mathrm{C}$ temperature range is initiated. The $600^{\circ} \mathrm{C}$ temperature is indicated to be the minimum temperature at which the helium release mechanism operating in the $600-1300^{\circ} \mathrm{C}$ temperature range is measurably observed in the present experiments.

The burst-type helium release in experiment 3 occurred over the 300 to $1100^{\circ} \mathrm{C}$ temperature range. This type of heljum release decreased as time progressed in the $800^{\circ} \mathrm{C}$ steady-state helium release experiment; it increased with increasing time in the 500 and $650^{\circ} \mathrm{C}$ steady-state experiments. The results from these experiments show that the burst-type helium release is related to the helium concentration in the sample over the 300 to $1100^{\circ} \mathrm{C}$ temperature range and is the major helium release mechanism operating below the $650^{\circ} \mathrm{C}$ temperature range.

As shown by the gradual disappearance of the burst-type helium release in the $800^{\circ} \mathrm{C}$ experiment, this type of release becomes secondary during steadystate experiments above the $650^{\circ} \mathrm{C}$ temperature range.

The gradual increase in the burst-type helium release at temperatures below $650^{\circ} \mathrm{C}$ indicates that as the concentration of helium increases in a radiation damaged fuel form the single helium atoms form clusters. As time progresses the concentration of the clusters increase such that they interact in providing an interconnected microstructure through which the clusters may be released. Above the $650^{\circ} \mathrm{C}$ temperature range, the experiments indicate that the clusters or dislocation loops formed by radiation damage become significantly more mobile and migrate to releasing areas. The rate of annealing for this process at temperatures greater than $650^{\circ} \mathrm{C}$ is such that coalescence of large numbers of helium-filled clusters or dislocation loops is effectively reduced. The helium release rate becomes more uniform with the burst-type helium release almost eliminated as the sample's temperature is maintained for long periods of time at temperatures above $650^{\circ} \mathrm{C}$.

Vapor Pressure (J. C. Posey)

The vapor pressure of $\mathrm{Cm}_{2} \mathrm{O}_{3}$ is being measured using the Knudsen cell method. Several preliminary values have been determined and compared to the earlier work of Smith and Peterson. ${ }^{7}$ Current work has been directed primarily toward the elimination of possible systematic exrors and the reduction of random error. The data determined so far in the current work are shown in Fig. 5 along with a part of the data of Smith and Peterson. The two groups of data can be compared at about $1600^{\circ} \mathrm{C}$ where their temperature ranges touch. The ORNL data are lower than those determined by Smith and Peterson in Run 2 but agree well with those determined in Run 1 . Smith and Peterson, however, believed the Run 2 data were their best because of the presence of about 12.5 mole \% impurities in the $\mathrm{Cm}_{2} \mathrm{O}_{3}$ used in Run 1 . 
They also conducted two other runs which gave badly scattered higher data. Our methods are being examined for possible systematic error.

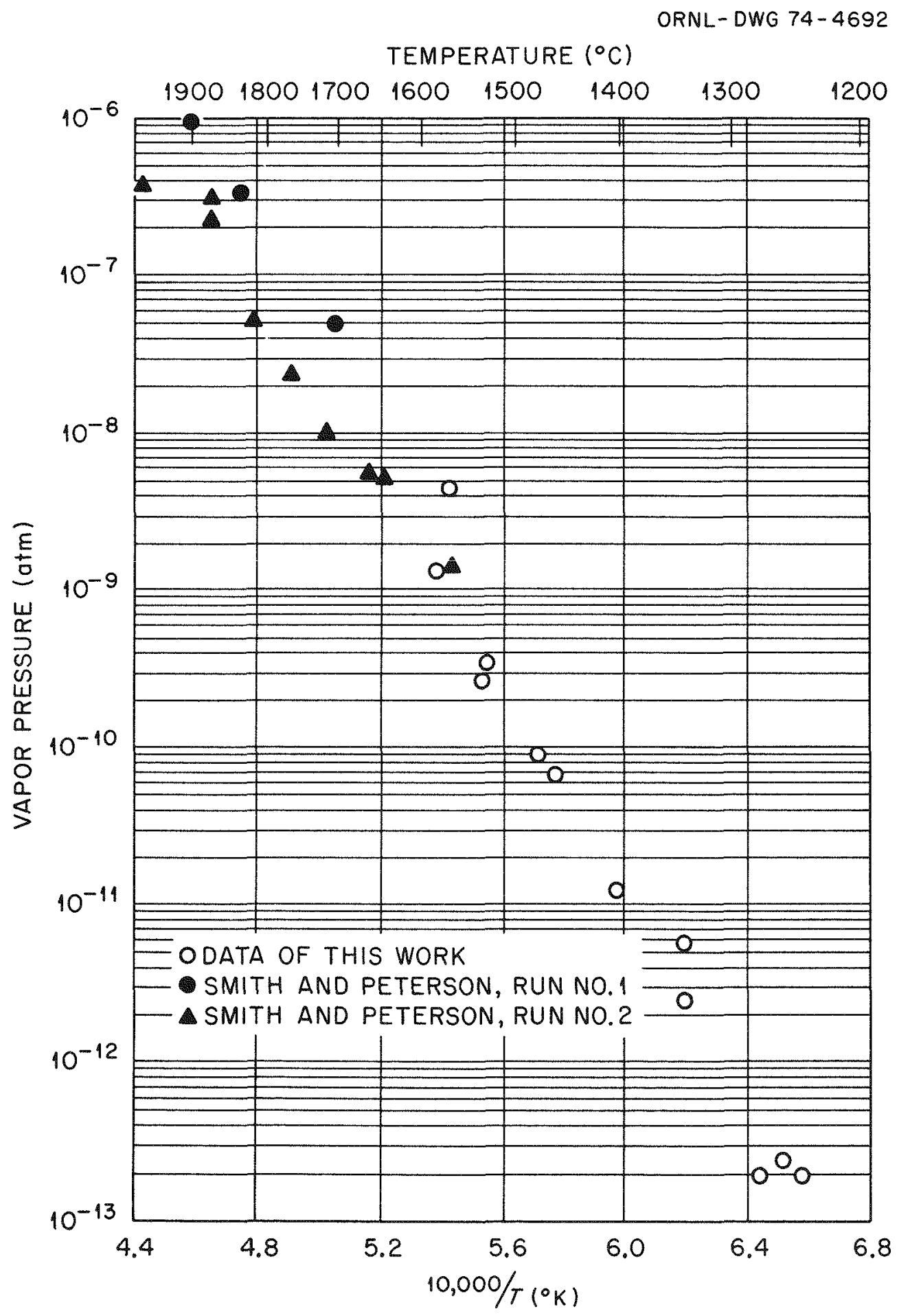

Fig. 5. Vapor Pressure of $\mathrm{Cm}_{2} \mathrm{O}_{3}$. 
All of the data are presented as if the $\mathrm{Cm}_{2} \mathrm{O}_{3}$ were present in the vapor state as $\mathrm{Cm}_{2} \mathrm{O}_{3}$ molecules. (It is necessary to assume a molecular weight to calculate vapor pressure from Knudsen cell data.) Smith and Peterson presented their data in this form because the actual vapor species had not been proven. They, however, deduced from thermodynamic considerations that $\mathrm{Cm}_{2} \mathrm{O}_{3}$ probably vaporized according to the following disproportionation reaction:

$$
\mathrm{Cm}_{2} \mathrm{O}_{3} \rightleftharpoons 2 \mathrm{Cmo}+\mathrm{O}
$$

Plans call for the determination of the vapor species by mass spectrometer in the future.

In our work a known fraction of the $\mathrm{Cm}_{2} \mathrm{O}_{3}$ leaving the Knudsen cell impinges on a target plate at the top of the furnace. The amount of curium on the target is determined by an alpha counting procedure, and the amount of $\mathrm{Cm}_{2} \mathrm{O}_{3}$ leaving the Knudsen cell is calculated from this value. This calculation assumes that all of the molecules striking the target stick, but this could not be assumed with certainty. In some similar reported cases, the condensation coefficient is much less than one.8,9 (The condensation coefficient is the fraction of the molecules striking the surface which stick.) The size of the condensation coefficient is strongly influenced by the nature of the surface. High values occur when the condensing molecules readily fit into the crystalline lattice of the surface.

Several targets with differing surfaces were prepared and exposed in the vapor pressure apparatus. The results are given in Table 25 .

Table 25. Effect of the Nature of Target Surface on the Amount of Curium-244 Collected

\begin{tabular}{|c|c|c|c|}
\hline $\begin{array}{l}\text { Exposure } \\
\text { Temperature } \\
\left({ }^{\circ} \mathrm{C}\right)\end{array}$ & $\begin{array}{l}\text { Exposure } \\
\text { Time } \\
(h r)\end{array}$ & $\begin{array}{c}\text { Curium-244 } \\
\text { Deposited } \\
(\mu g)\end{array}$ & Nature of Surface \\
\hline 1535 & 0.500 & $10.9 \times 10^{-5}$ & Stainless steel \\
\hline 1535 & 0.500 & $9.84 \times 10^{-5}$ & Oxidized stainless steel \\
\hline 1535 & 0.500 & $9.35 \times 10^{-5}$ & $\mathrm{Nd}_{2} \mathrm{O}_{3}$ deposited on stainless steel \\
\hline 1535 & 0.500 & $8.29 \times 10^{-5}$ & $\begin{array}{l}\text { Lead electroplated on stainless } \\
\text { steel and lightly oxidized }\end{array}$ \\
\hline 1535 & 0.500 & $9.27 \times 10^{-5}$ & Platinum \\
\hline Average & deposit & $9.53 \times 10^{-5}$ & $s(x)=0.95 \times 10^{-5}$ \\
\hline 1492 & 1.00 & $7.80 \times 10^{-5}$ & Stainless steel \\
\hline 1490 & 1.00 & $7.32 \times 10^{-5}$ & Oxidized stainless steel \\
\hline 1490 & 1.00 & $7.80 \times 10^{-5}$ & $\mathrm{Nd}_{2} \mathrm{O}_{3}$ deposited on stainless steel \\
\hline 1492 & 1.00 & $8.70 \times 10^{-5}$ & $\begin{array}{l}\text { Lead electroplated on stainless } \\
\text { steel and lightly oxidized }\end{array}$ \\
\hline Average & deposit & $7.90 \times 10^{-5}$ & $S(x)=0.58 \times 10^{-5}$ \\
\hline
\end{tabular}


Any influence of surface composition is entirely obscured by the random scatter of the data. The standard deviation of points about the mean was $0.95 \times 10^{-5} \mu \mathrm{g}$ or $10 \%$ for the $1535^{\circ} \mathrm{C}$ data and $0.58 \times 10^{-5} \mu \mathrm{g}$ or $7.3 \%$ for the $1490^{\circ} \mathrm{C}$ data. Stainless steel gave the highest value in the $1535^{\circ} \mathrm{C}$ tests but was below average in the $1490^{\circ} \mathrm{C}$ test; lead gave the lowest value in the $1535^{\circ} \mathrm{C}$ test but gave the highest value in the $1490^{\circ} \mathrm{C}$ test. It was concluded that the condensation coefficient is little influenced by the nature or the composition of the surface of the target. It is not likely that five different substances would exhibit essentially equal condensation coefficients unless the coefficients were all near unity. The point could be proven rigorously by means of a hollow target which would operate in a manner analogous to a black body hole as used in the measurement of temperature by optical pyrometer. Since this proof would involve substantial time and expense, it is not planned.

The original temperature control equipment functioned poorly in the lower temperature range. The temperature tended to drift in an erratic manner. This made the long runs required at the lower temperatures nearly impossible and limited the temperature range. A proportional controller was designed, fabricated, and installed on the furnace power supply. The controller is different from the previous one in that it maintains a desired constant power input to the furnace whereas the previous one maintained a constant current input to the furnace. The present controller can also linearly ramp the power level from any two positions over the entire furnace temperature range, and the ramping time can be varied linearly from one-half to six hours. The present controller should maintain a more constant operating temperature over the complete temperature range. In an experiment with the new controller at a furnace temperature of $1260^{\circ} \mathrm{C}$, the temperature was maintained essentially constant over a 72-hr period.

In order to test the temperature uniformity of the Knudsen cell used in the $\mathrm{Cm}_{2} \mathrm{O}_{3}$ vapor pressure experiments, a second Knudsen cell of identical physical dimensions has been placed in the glove box. This second cell has two additional black body holes; one located immediately above the present one on the base and the other located on the upper piece near the top surface. The temperature calibrations will show if any thermal gradients exist along the cell. If a significant temperature difference is found, the cell can be oriented such that the temperature difference will be minimized.

In preparation for a re-run on several data points of the vapor pressure, new target holders were fabricated. The use of a fresh target holder for each cycle of measurements should reduce the likelihood of incidental contamination on the targets; this is especially important at the lowtemperature end of the range since the amount of ${ }^{244} \mathrm{Cm}$ collected is so sma11. In combination with the improved temperature control reported last month, this technique should provide better data. 
Dimensional Stability (C. I. Ottinger)

The 25-W pellet of purified $\mathrm{Cm}_{2} \mathrm{O}_{3}$ (PCM-P1) which had been exposed to dry air for $\sim 4$ months was broken up in order to use the ${ }^{244} \mathrm{Cm}_{2} \mathrm{O}_{3}$ for other experiments. Although the material which had flaked off from the ends of this pellet was a coarse powder, the remaining bulk pellet was quite hard and difficult to fracture. It was finally broken up by placing it in a cylindrical well of a chill block and using a striking rod to impact the pellet. The other purified ${ }^{244} \mathrm{Cm}_{2} \mathrm{O}_{3}$ pellet (PCM-P2), which had been stored under argon, was exposed to dry air and observed. In contrast to PCM-P1, there was little flaking of material from the edges of this pellet during the first few days. However, multiple small cracks appeared in the edge zones, indicating that a similar phenomenon was occurring. Observation of this pellet will continue.

Solubility of Stored Curium Oxide Powder

in Air-Saturated Distilled Water (J. C. Posey)

A test was made of the solution behavior of curium oxide powder from the stored inventory of curium oxide. This powder has a composition falling between ${ }^{244} \mathrm{Cm}_{2} \mathrm{O}_{3}$ and ${ }^{244} \mathrm{CmO}_{2}$. A 0.0858 -g sample of the powder was placed in $150 \mathrm{ml}$ of distilled water contained in a stainless steel beaker. The beaker was covered to reduce evaporation. The mixture was agitated by a teflon covered magnetic stirrer. The heat of decay of the ${ }^{244} \mathrm{Cm}$ and the energy of stirring caused the temperature to be $38^{\circ} \mathrm{C}$ although the room temperature was approximately $25^{\circ} \mathrm{C}$. Samples were withdrawn at intervals through $10-$ to $20-\mu \mathrm{m}$ sintered glass filter sticks and their ${ }^{244} \mathrm{Cm}$ contents were determined by an alpha counting procedure.

The solution concentration as a function of time is shown in Fig. 6 . The reason for the series of peaks is not known. It is possible that different chemical compounds were formed as time progressed. Curium forms four known anhydrous oxides, $\mathrm{CmO}_{1.5}, \mathrm{CmO}_{1.71}, \mathrm{CmO}_{1.83}$, and $\mathrm{CmO}_{2}$. Little is known concerning the hydrated oxides or hydroxides.

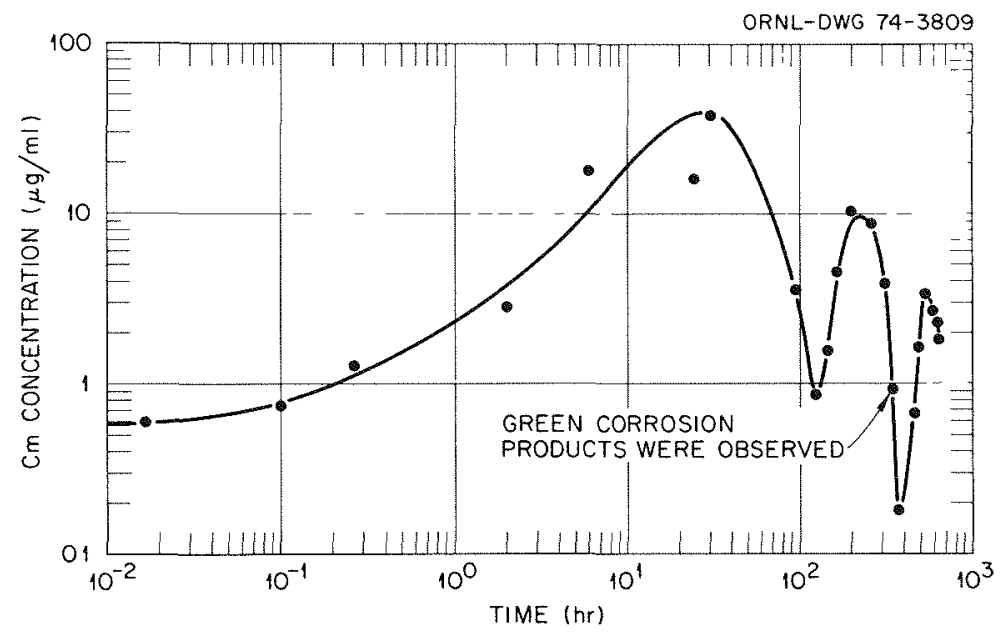

Fig. 6. Solution of $\mathrm{CmO}_{\mathrm{x}}$. 
At $338 \mathrm{hr}$, an olive green color was observed and the volume of powder appeared to increase. This may have been caused by corrosion of the beaker.

Criticality of ${ }^{244} \mathrm{Cm}_{2} \mathrm{O}_{3}$ Fuels (T. A. Butzer and $K$. W. Haff)

In a previous report ${ }^{l}$ the calculated abundances of the principal isotopes of curium were shown to vary depending on the type of commercial power reactor and composition of the fuel. The abundance of the sum of the fissile isotopes $243 \mathrm{Cm}$ and ${ }^{245} \mathrm{Cm}$ varied in the range 6.4 to 12.2 atom \%. This contrasts with $<1$ atom \% of the fissile isotopes in Savannah River product ${ }^{244} \mathrm{Cm}$, which is currently being used for fuel characterization, and raised the question of criticality safety of some heat source designs. The following criticality evaluation was prepared by the ORNL Neutron Physics Division.

A study of the criticality parameters of three ${ }^{244} \mathrm{Cm}$-fueled heat sources using the KENO Monte Carlo code has been made. Heat source designs considered, designated $A, B$, and $C$, are described in Table 26. Cross sections used were from the 100 group set processed from data in the ENDF/B data file, except those for ${ }^{245} \mathrm{Cm}$, which were compiled by 0 . L. Smith as part of a study for the ORNL Reactor Division. A11 calculations assumed water or graphite between units in the arrays studied and $15-\mathrm{cm}$ water reflection external to the arrays. Curium isotope abundances were taken from those for PWR (U $+\mathrm{Pu}$ ) fuel, since the highest percent $(12.2 \%)$ of $245 \mathrm{Cm}$ occurred here. Cross sections for ${ }^{244} \mathrm{Cm}$ and $245 \mathrm{Cm}$ only were used in the calculation. Curium-243 was included with $245 \mathrm{~cm}$, and $246 \mathrm{~cm}$ was included with $244 \mathrm{~cm}$. Because of the smal1 amounts of $243 \mathrm{Cm}$ and $246 \mathrm{~cm}$ present, this approximation should not introduce any significant error, and based on available data, it should result in a high estimate of $k_{\text {eff }}$.

Table 26. Curium-244 Heat Source Descriptions

\begin{tabular}{|c|c|c|c|}
\hline Design & Unit Description & $\begin{array}{l}\text { No, of } \\
\text { Units }\end{array}$ & $\begin{array}{l}\text { Total } \\
\text { Watts }\end{array}$ \\
\hline$A$ & $\begin{array}{l}\text { Fourteen }{ }^{244} \mathrm{Cm}_{2} \mathrm{O}_{3} \text { pellets totaling } 400 \mathrm{~W} \text {; } \\
\text { arranged uniformily within a graphite } \\
\text { sphere; encapsulated in metal and graphite } \\
\text { concentric shells; overall diameter } 3.5 \mathrm{in} \text {. }\end{array}$ & 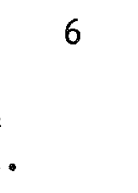 & 2400 \\
\hline $\mathrm{B}$ & $\begin{array}{l}\text { Two pencil-type }{ }^{244} \mathrm{Cm}_{2} \mathrm{O}_{3} \text { sources in thin } \\
\text { metal capsules totaling } 300 \mathrm{~W} \text {; arranged } \\
\text { side-by-side in a rectangular graphite } \\
\text { body } 3 \times 3 \times 1.5 \mathrm{in} .\end{array}$ & 28 & 8400 \\
\hline $\mathrm{C}$ & $\begin{array}{l}{ }^{244} \mathrm{Cm}_{2} \mathrm{O}_{3} \text { pellets totaling } 4400 \mathrm{~W} \text { stacked } \\
\text { to form a column } 1.1 \text {-in. diameter by } 15 \text { - } \\
\text { in. high; encapsulated in metal cladding } \\
\text { (no graphite) }\end{array}$ & 2 & 8800 \\
\hline
\end{tabular}


For all the systems studied, the geometries were represented as accurately as was deemed reasonable for a survey study. Any approximations made were made so as to increase the multiplication of the systems.

The effect of resonance corrections for ${ }^{244} \mathrm{Cm}$ and of various cross section compilations for ${ }^{245} \mathrm{Cm}$ was considered. The effect of these two factors was found not to be significant for these systems.

$\underline{\text { Design A }}$

Source design A was approximated by three concentric spherical annular regions consisting of void, homogenized fuel with graphite, and a region of low density graphite. Several thin layers consisting of Haynes 188, TZM, and graphite surrounded this core. A $2 \times 2 \times 2$ water-moderated array was used, with six of the units containing spheres and with the outside layers in contact, and the other two units containing water. This arrangement resulted in a $k_{\text {eff }}$ of $0.234 \pm 0.005$.

In order to test how well this configuration represented the actual geometry, another geometry was used in which the fuel-graphite region was divided into two regions, one with graphite only and the other with higher than average density fuel mixed with the rest of the graphite. This new arrangement more closely approximates the actual geometry of the sphere and gave a $k_{\text {eff }}$ of $0.233 \pm 0.005$, indicating no significant effect.

It was noted during the course of the calculations that the fission cross sections for the ${ }^{245} \mathrm{Cm}$ set generated at ORNL were somewhat different from a set which had been compiled at the Savannah River Plant. In view of possible uncertainties in these cross sections, a new set was generated which contained the higher value of fission cross sections from these two sets for all energies. Scattering and capture cross sections from the ORNL set were used. Such a set should signficantly reduce the possibility of underestimating the criticality of the $244 \mathrm{~cm}$ heat sources. The use of this cross-section set for the spheres described above gave a $k_{\text {eff }}$ of $0.244 \pm 0.005$, which is only a relatively small increase.

\section{$\underline{\text { Design B }}$}

Source design $B$ was represented by a $3 \times 3 \times 4$ water-moderated array, with the bottom $3 \times 3$ (27 units) and one central unit at the top containing fuel pellets. The remaining eight units on the top contained water. For no separation between units, this arrangement gave a $k_{\text {eff }}$ of $0.402 \pm 0.005$. In order to test the effect of varying amounts of water between the units, the edge-to-edge spacing was varied by $1-\mathrm{cm}$ increments. It was found that the highest $k_{\text {eff }}$, occurring at $2-\mathrm{cm}$ separations, was $0.441 \pm 0.008$.

In the above calculations, no allowance had been made for the effect of self-shielding in the resonance capture cross sections of $244 \mathrm{Cm}$. An additional calculation at 2-cm separations which included the effects of the resonance corrections gave a $k_{\text {eff }}$ of $0.453 \pm 0.008$, which is only a small increase over the previous result. In addition, a calculation 
which used the modified fission cross section described previously, as well as the resonance correction, yielded a $k_{\text {eff }}$ of $0.471 \pm 0.007$. This is the highest $k_{\text {eff }}$ obtained for any of the calculations in this study.

Since all of the units contain graphite, the possibility of graphite moderation between elements of the array was considered. The arrays were water reflected. Design B was chosen for this study, since it had the highest $k_{\text {eff }}$ for water moderation. For various spacings between the units, the highest value of $k_{\text {eff }}$ with graphite moderation was $0.421 \pm$ 0.006 at $0-\mathrm{cm}$ separation between units.

Design C

Source design $C$ consisted of two adjacent rods of fuel, with several layers of various cladding. This arrangement, with water moderation, resulted in a $k_{\text {eff }}$ of $0.331 \pm 0.007$.

Summary of Results

The results of $244 \mathrm{Cm}$ heat source calculations are summarized in Table 27. The effect of varying the separations between adjacent units is demonstrated in the design $B$ values. Since the $k_{\text {eff }}$ values here are higher than for the $A$ and $C$ designs, it appears unlikely that the highest $k_{\text {eff }}$ for design $B$ results will be exceeded for any spacing of the other designs. If the maximum number of units are not exceeded and no other major changes in design are encountered, the heat sources appear to have no criticality problem.

Table 27. Results of Criticality Evaluation of Curium-244 Sources

\begin{tabular}{|c|c|c|c|c|}
\hline Design & $\begin{array}{l}\text { No. of } \\
\text { Units }\end{array}$ & $\begin{array}{l}\text { Edge-to-Edge } \\
\text { Spacing }(\mathrm{cm})\end{array}$ & $k_{\text {eff }}$ & Remarks \\
\hline$A^{a}$ & 6 & $\begin{array}{l}0 \\
0 \\
0\end{array}$ & $\begin{array}{l}0.234 \pm 0.005 \\
0.233 \pm 0.005 \\
0.244 \pm 0.005\end{array}$ & $\begin{array}{l}\text { Extra region in sphere } \\
\text { Revised } 245 \mathrm{Cm} \text { fission cross sections }\end{array}$ \\
\hline $\mathrm{B}^{\mathrm{a}}$ & 28 & $\begin{array}{l}0 \\
1 \\
2 \\
2 \\
2 \\
3\end{array}$ & $\begin{array}{l}0.402 \pm 0.005 \\
0.431 \pm 0.007 \\
0.441 \pm 0.008 \\
0.453 \pm 0.008 \\
0.471 \pm 0.007 \\
0.405 \pm 0.008\end{array}$ & $\begin{array}{l}\text { Resonance corrected } 244 \mathrm{Cm} \\
\text { Resonance corrected } 244 \mathrm{Cm} \text { and } \\
\text { revised } 245 \mathrm{Cm} \text { fission cross sections }\end{array}$ \\
\hline $\mathrm{B}^{\mathrm{b}}$ & 28 & $\begin{array}{l}0 \\
1 \\
3 \\
5\end{array}$ & $\begin{array}{l}0.421 \pm 0.006 \\
0.399 \pm 0.006 \\
0.353 \pm 0.006 \\
0.334 \pm 0.007\end{array}$ & $\begin{array}{l}\text { Resonance corrected } 244 \mathrm{Cm} \text { and } \\
\text { revised } 245 \mathrm{Cm} \text { fission cross sections } \\
\text { Same } \\
\text { Same } \\
\text { Same }\end{array}$ \\
\hline$c^{a}$ & 2 & 0 & $0.331 \pm 0.007$ & \\
\hline
\end{tabular}

Water-moderated, water-reflected.

${ }^{b}$ Graphite-moderated, water-reflected. 
Effects of Plutonium In-Growth (C. L. Ottinger)

Current experimental work with $244 \mathrm{Cm}$ utilizes for the most part "stock" ${ }^{244} \mathrm{Cm}$ oxide resulting from a processing campaign carried out at SRL during the period 1968-1971. A small fraction of $24{ }^{4} \mathrm{Cm}$ was recovered from waste residues at ORNL in 1973 to provide some low-plutonium material for experiments, but except for this fraction materials available contain plutonium concentrations ranging upwards from $\sim 10 \%$. Although these are referred to as "production-grade," this term is not intended to imply that they are identical to the ${ }^{244} \mathrm{Cm}$ products that would be expected from a by-product $2{ }^{24} \mathrm{Cm}$ recovery plant.

Among the as yet unanswered questions in the development of ${ }^{244} \mathrm{Cm}$ for heat source use are those related to the effects of impurities. The impurities which will be present in practical ${ }^{244} \mathrm{Cm}$ fuels are of two types - process contaminants and plutonium. Process contaminants are those which are not totally removed during the ${ }^{244} \mathrm{Cm}$ separations process or which are introduced as a result of the process; these may include any of a large variety of impurities at various levels. Since the development of process flowsheets is still only in the study stage, evaluation of the likely process impurities can be only an educated guess at present. Based on previous processing experience, it can be inferred that americium will be the primary process contaminant, and that it will be present in concentrations of about 1-2 wt \%. For study purposes, an initial ratio of process impurities to curium of 0.03 has been assumed, but no assumed distribution of impurities has been established. Possible influences of process impurities include effects on fabricability of fuels to compatibility, vaporization characteristics, thermophysical properties, and others in addition to the obvious effect on power density. It will be necessary to evaluate these effects both by simulation and eventually by practical demonstration when typical fuels are available.

While the types and amounts of process impurities cannot be accurately predicted at present, the amount of plutonium can be since it varies only with the time since separation of the ${ }^{244} \mathrm{Cm}$. (It can be confidently assumed that the plutonium content will be insignificant as of separation time.) In any processing/fuel fabrication/source encapsulation system which includes a few months of storage and/or shipment time for the products, plutonium will be the major contaminant in the fuel as of time of source fabrication. Also, the "curium" materials used in experimental work are of course mixtures of curium and plutonium (at least), and the interpretation of experimental results must take this into account. Not only the concentration of plutonium but also its chemical form can influence the characteristics of the fuel.

It has been generally assumed that a ${ }^{244} \mathrm{Cm}$ recovery system would process the ${ }^{244} \mathrm{Cm}$ to the $\mathrm{CmO}_{2}$ form for storage and that it would be converted to the $\mathrm{Cm}_{2} \mathrm{O}_{3}$ form immediately prior to encapsulation. During the period between chemical separation and encapsulation, any plutonium resulting from decay would thus be in the dioxide form. The procedures normally used for conversion of the $\mathrm{CmO}_{2}$ to $\mathrm{Cm}_{2} \mathrm{O}_{3}$ (typically calcination at $1000^{\circ} \mathrm{C}$ in an oxygen-free atmosphere) will not convert the $\mathrm{PuO}_{2}$ to the 
sesquioxide form. As of the time of encapsulation, the fuel will be composed of $\mathrm{Cm}_{2} \mathrm{O}_{3}$ and $\mathrm{PuO}_{2}$ plus the oxides of the process impurities. After encapsulation there will be no free oxygen available for oxidation of the decay-produced plutonium, and the mixed oxide system will become more complex.

One approach to dealing with the question of the chemical form of the decay-produced plutonium would be to establish curium process systems to provide $\mathrm{Cm}_{2} \mathrm{O}_{3}$ as the product form. The $\mathrm{Cm}_{2} \mathrm{O}_{3}$ could be stored under oxygen-free conditions and all subsequent operations carried out in inert-atmosphere enclosures. Both the curium and the decay-produced plutonium oxides would then remain in the most reduced state. While the $\mathrm{Pu}_{2} \mathrm{O}_{3}$ may exert significant influences on the characteristics of the fuel, the types and magnitudes of such influences could be determined systematically. Also, thermodynamic calculations indicate that $\mathrm{PuO}_{2}$ may react with components of several potential encapsulants, so elimination of $\mathrm{PuO}_{2}$ from the fuel may be attractive in this regard.

The recent re-orientation of the ${ }^{244} \mathrm{Cm}$ development program requires that most of the ${ }^{244} \mathrm{Cm}$ now in experimental facilities be encapsulated for long-term storage. These materials include batches ranging in plutonium content from less than $5 \%$ to nearly $20 \%$. Selected fractions will be converted to the $\mathrm{Cm}_{2} \mathrm{O}_{3}$ form prior to encapsulation so that samples will be available in the future for examination.

\section{General Operations (C. L. Ottinger)}

The furnace and associated in-cell equipment which had been used for ceramic compatibility work were disassembled and discarded. Cell services were removed and decontamination was started in order to release the cell for other operations. A considerable quantity of solid waste was removed from the Curium Souce Fabrication Facility cells and the equipment was checked out in preparation for ${ }^{244} \mathrm{Cm}_{2} \mathrm{O}_{3}$ pelletization work.

\section{Curium-244 Recovery Development

$$
\text { (C. L. Ottinger and J. C. Posey) }
$$

A program has been initiated to investigate various processes having potential applicability for the recovery of ${ }^{244} \mathrm{Cm}$ from the wastes resulting from processing of power reactor fuels. The ultimate goal of this program is the demonstration of one or more flowsheets adaptable to production-scale processing. If ${ }^{244} \mathrm{~cm}$ is to be utilized in significant quantities for power generation or other applications, a system must be developed which is attractive when compared to alternative sources of power. Comparisons between $244 \mathrm{Cm}$ power sources and other potential sources will be based on several criteria, including relative safety, applicability, system weights (for space applications), availability, and, of course, cost. The cost of recovery and purification will be a significant factor in any analysis, and process development information will be required in order to predict these costs. 
Since any ${ }^{244} \mathrm{Cm}$ recovery process must eventually be incorporated into a system which includes not only the separations process itself, but also receipt of feed material and preparation of solid wastes for storage, certain limitations are imposed on the $244 \mathrm{~cm}$ process. In addition to meeting the normal criteria of technical feasibility, safety, and economics the process must be designed to limit the volume of solid waste to the lowest practical value. The process should also be as versatile as possible in order to accomodate changes in feed composition. Future feed supplies (i.e., reactor fuel wastes) are expected to fall into two general categories: 1) feeds containing essentially fission products with relatively small amounts of other contaminants; and 2) feeds in which a large fraction of the elemental content results from process chemicals added during fuel reprocessing operations. Two reference fuel compositions will be defined to represent these two categories, and the necessary modifications to processes related to this variation will be investigated.

Processing of reactor fuel wastes for recovery of $24{ }^{4} \mathrm{Cm}$ will involve four distinct but inter-related phases:

1. Feed Conditioning: Modification of the stored reactor fuel waste to provide a solution ready for ${ }^{244} \mathrm{Cm}$ processing.

2. LN/AN Separation: Processing to provide a ${ }^{244} \mathrm{Cm}$ stream which is composed essentially of those lanthanides (LN) and actinides (AN) that are analogs of curium in classical processes.

3. AN Separation: Fractionation of the LN/AN stream to provide a ${ }^{244} \mathrm{Cm}$ stream containing americium as the primary contaminant.

4. Purification: Final separation of the ${ }^{244} \mathrm{Cm}$ from the americium and the remaining other contaminants.

Each of these phases may incorporate several unit processes or operations, but this division provides a convenient description of the required group operations. The initial area of concentrated effort in this program will be Phase 3, although some work will be done on other phases concurrently. Solvent extraction, in particular differential extraction, appears to offer several advantages as applied to the separation of actinides from lanthanides, and this process will be evaluated. 


\section{REFERENCES}

1. Eugene Lamb, ORNL Isotopic Power Fuels Quarterly Report for Period Ending September 30, 1973, ORNL-4932, Oak Ridge National Laboratory.

2. Alvin Glassner, The Thermochemical Properties of the Oxides, Fluorides and ChZorides to $2500^{\circ} \mathrm{K}$, ANL-5750, Argonne National Laboratory (1957).

3. F. L. Oetting, "The Chemical Thermodynamic Properties of P1utonium Compounds," Chem. Rev. 67, 261-97 (June 1967).

4. O. Kubaschewski, E. L. Evans, and C. B. Alcock, MetalZurgical Thermochemistry, Pergamon Press, New York, 1967, pp. 421-29.

5. K. A. Gschneidner, Rare Earth Alloys, D. Van Nostrand Company, Inc., Princeton, N. J., 1961, p. 237.

6. P. Angelini and R. E. McHenry, Helium Release from ${ }^{244} \mathrm{Cm}_{2} \mathrm{O}_{3}$, ORNL-4785, Oak Ridge National Laboratory (November 1973).

7. P. K. Smith and D. E. Peterson, "High-Temperature Evaporation and Thermodynamic Properties of $\mathrm{Cm}_{2} \mathrm{O}_{3}, " \mathrm{~J}$. Chem. Phys. 52(10), 4963-72 (May 1967).

8. John L. Margrave, Physicochemical Measurements at High Temperatures, J. O'M. Bockris, J. L. White, and J. D. McKenzie (eds.), Butterworths Scientific Publications, London, 1959, pp. 230-31.

9. Saul Dushman and J. M. Lafferty, Scientific Foundations of Vacuum Technique, 2nd Ed., John Wiley \& Sons, Inc., New York, 1962, pp. 408-10. 


\section{Blank page}


ORNL -4978

UC-33 - Nuclear Propulsion

Systems and Aerospace Safety

INTERNAL DISTRIBUTION

1-3. Centra1 Research Library

4. ORNL - Y-12 Technical Library

Document Reference Section

5-24. Laboratory Records Department

25. Laboratory Records, ORNL RC

26. G. M. Adamson, $J r$.

27. P. Angelini

28. N. C. Bradley

29. T. A. Butler

30. C. E. Clifford

31. F. L. Culler

32. J. E. Cunningham

33. J. R. DiStefano

34. R. G. Donnelly

35. D. E. Ferguson

36. J. H. Gillette

37. K. W. Haff
38. R. F. Hibbs

39. M. R. Hill

40. H. Inouye

41. E. Lamb

42. R. E. Leuze

43. H. C. McCurdy

44. C. L. Ottinger

45. Herman Postma

46. J. C. Posey

47. A. F. Rupp

48. R. W. Schaich

49. M. K. Skinner

50. D. A. Sundberg

51. D. B. Trauger

52. J. R. Weir

53. A. Zucker

\section{EXTERNAL DISTRIBUT ION}

54. R. D. Baker, Los Alamos Scientific Laboratory

55. F. P. Baranowski, Division of Production \& Materials Management, AEC

56. M. Bel1, TRW Systems

57. Linda Benker, Thermo Electron Corporation

58. R. F. Borlick, Division of Production \& Materials Management, AEC

59. H. C. Carney, Jr., Gulf Energy and Environmental Services

60. R. T. Carpenter, Division of Space Nuclear Systems, AEC

61. W. T. Cave, Mound Laboratory

62. Commander, Naval Undersea Research and Development Center

63. G. R. Crane, Headquarters AFSC (XRTS), Andrews Air Force Base

64. G. P. Dix, Division of Space Nuclear Systems, AEC

65. R. English, NASA, Lewis Research Center

66. T. P. Fleming, Naval Facilities Engineering Command

67. R. K. Flitcraft, Mound Laboratory

68-70. E. E. Fowler, Division of Applied Technology, AEC

71. L. Frank, Weiner Associates, Inc.

72. A. G. Fremling, AEC-Richland Operations office

73. D. S. Gabrie1, Division of Space Nuclear Systens, AEC

74. N. Goldenberg, Division of Space Nuclear Systems, AEC

75. M. Goldman, University of California

76. R. C. Hamilton, IDA - Room 19, 400 Army-Navy Drive, Arlington, VA

77. W. J. Haubach, Division of Physical Research, AEC

78. M. G. Hegarty, Radio Corporation of America

79. T. B. Hindman, AEC-Savannah River Operations Office

80. R. E. Hopkins, U.S. Army Mobility Equipment R\&D Center 
81. Ian Jones, TRW Systems

82. R. J. Katucki, General Electric

83. T. B. Kerr, NASA Headquarters

84. F. E. Kruesi, Savannah River Laboratory

85. G. Linkous, Teledyne-Isotopes, Inc.

86. W. A. McDona1d, Teledyne-Isotopes, Inc.

87. T. A. Nemzek, Division of Reactor Research and Development, AEC

88. G. A. Newby, Division of Space Nuclear Systems, AEC

89. W. G. Parker, Westinghouse Astronuclear Laboratory

90. F. K. Pittman, Division of Waste Management \& Transportation, AEC

91. G. B. Pleat, Division of Production \& Materials Management, AEC

92. R. W. Ramsey, Jr., Division of Waste Management \& Transportation, AEC

93. P. K. Smith, E. I. duPont-Savannah River Laboratory

94. J. M. Teem, Division of Physical Research, AEC

95. H. H. Van Tuy1, Pacific Northwest Laboratories

96. R. L. Wainwright, Dayton Area office, AEC

97. J. L. Womack, E. I. duPont-Savannah River Plant

98. M. E. Wrenn, Division of Biomedical and Environmental Research, AEC

99. AEC, Richland Operations Office

100. AEC', Savannah River Operations office

101. General Electric Company, MSD, AEC

102. Genera1 Electric Company, VNC, AEC

103. NASA, Langley Research Center

104. NASA, Manned Spacecraft Center

105. NASA, Marshal1 Space Flight Center

106. Navy Space Systems Activity

107. Teledyne-Isotopes, Inc., Timonium, MD

108. Research and Technical Support Division, AEC, ORO

109. Patent office, AEC, ORO

110-280. Given Distribution as Shown in TID-4500 Under Nuclear Propulsion Systems and Aerospace Safety Category (25 copies - NTIS) 